\title{
Fritz Kahns Das Leben des Menschen
}

\section{Zur Produktion und Transkription eines populären Werks*}

\author{
Miriam Eilers
}

\begin{abstract}
Fritz Kahn's The Life of Man: Production and Transcription of a Bestseller
This paper investigates the production and circulation of the illustrations in Fritz Kahn's five-volume series The Life of Man, one of the most popular medical publications in the German interwar period. In 1912 Kahn (1888-1968), together with a staff of illustrators, began producing what would later become his best-selling series. Illustrations of this series (in particular, the poster The human factory/Der Mensch als Industriepalast) were widespread throughout Germany during the Weimar Republic. With the rise of National Socialism, Kahn was forced to emigrate in 1933. While his books were forbidden by the Nazi regime, their illustrations continued to play an important role in popular scientific discourse: they reappeared in the Nazi health-education programme and were adapted for Kahn's publications in exile at the same time. This paper discusses how their production process and collective authorship made this complex and heterogeneous reception possible.
\end{abstract}

Keywords: Popular medical book, production of popular scientific illustrations, science communication, health education, exile literature

\section{Das Leben des Menschen als Gegenstand eines transkriptiven Verfahrens}

Die populären medizinischen und naturwissenschaftlichen Veröffentlichungen des Mediziners und Autors Fritz Kahn (1888-1968) haben in den letzten Jahren das wissenschaftshistorische Interesse geweckt. Als sein bekanntestes Werk gilt Das Leben des Menschen, eine fünfbändige Buchreihe, die fast über den gesamten Zeitraum der Weimarer Republik von 1922-1931 erschien. Kahn wurde 1888 in Halle/Saale als Sohn des jüdischen Arztes und Schriftstellers Arthur Kahn geboren. Bis zu seinem Abitur im Jahr 1907 lebte er in Berlin und New York. In Berlin studierte er von 1907 bis 1912 Humanmedizin.

"Für Heyko Eilers (1951-2015). 
Nach dem nationalsozialistischen Machtwechsel emigrierte Kahn 1933 nach Palästina und 1941 nach New York. In den 1950er Jahren kehrte er nach Europa zurück und starb 1968 in der Schweiz.

Mehr als alle anderen wurde die Abbildung Der Mensch als Industriepalast (Abb. 1) im Zusammenhang mit technisierter Lebenswelt und medizinischer Aufklärung in der Weimarer Republik gezeigt (Sappol 2006; Borck 2007; Ahren and Sappol 2009) oder als Beispiel für den populären Gebrauch des Homunkulus angeführt (Draaisma 1999: 213). Obwohl es in der medizin- und wissenschaftshistorischen Literatur geradezu eine Renaissance des Kahn'schen Werkes gab, sind dessen historische Produktionsbedingungen bisher noch nicht im Detail untersucht worden. Die Frage nach den historischen Entstehungsbedingungen ist umso interessanter, da - wie auch in der Literatur schon häufig angemerkt wurde - eine Vielzahl von Abbildungsstilen

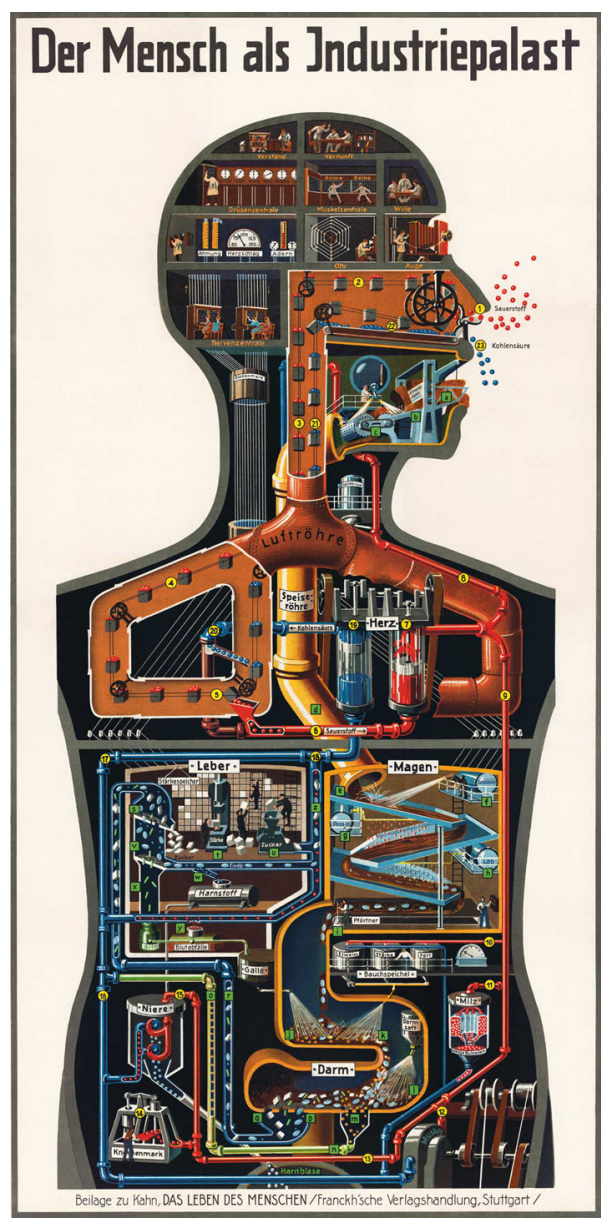

Abb. 1 "Der Mensch als Industriepalast” (Quelle: Plakat als Anlage zu „Das Leben des Menschen" Band 3, 1926. Copyright Franckh-Kosmos, Stuttgart) 
in Kahns Werk zu verzeichnen ist. Dies weist auf die Beteiligung einer Reihe von Illustratoren hin, wurde aber bisher Kahn selbst pauschal zugeschrieben, da die genauere Klärung der Autorschaft der Bilder ein Forschungsdesiderat darstellte (Borck 2007, 503). Anhand zugänglichen Archivmaterials untersucht dieser Beitrag die Zusammenarbeit zwischen Kahn und einigen der Illustratoren im konkreten Entstehungsprozess von Das Leben des Menschen. Dabei werden vor allem zwei Fragen aufgeworfen, die in der medizin- und wissenschaftsgeschichtlichen Literatur in den letzten Jahren diskutiert wurden, nämlich erstens die Frage nach der Rolle von Bildlichkeit im populären Wissen (Borck 2009) und zweitens die Frage nach Autorschaft im populären Wissensgenre (Azzouni 2015).

Kahns Buchreihe steht in der Tradition der Popularisierung der Naturwissenschaften. Deren Geschichte ist in den letzten Jahren intensiv untersucht worden (Daum 2002; Schwarz 2001). Hierbei stand insbesondere die Verbindung von Wissenschaft und Öffentlichkeit im Fokus (Nikolow and Bluma 2002; Nikolow and Bluma 2009). Während es in der Auseinandersetzung mit Populärwissenschaft oft um Fragen des Transfers und der Veränderung von Wissen in der Bewegung vom akademischen zum öffentlichen Raum ging, geht es mir hier darum zu zeigen, wie hier ein genuines Wissen mit eigenen Formen, Publikationsorganen und Sprachen entstehen konnte. Zur Bezeichnung dieser Wissensform verwende ich den Begriff ,populäres Wissen', wie er in neueren Studien in Abgrenzung zu ,populärer Wissenschaft' oder ,Populärwissenschaft' verwendet wird. Ina Heumann bezeichnet als ,populäres‘ jenes Wissen, das in allgemeinverständlichen Veröffentlichungen als Buch, Zeitschrift oder Bild erscheint (Heumann 2014: 24). Die konsequente Verwendung des Terminus ,populäres Wissen' ermöglicht es, die Transformationen dieses Wissens als epistemisch eigenständigen Vorgang (d.h. unabhängig von akademischem Wissen) zu analysieren und an das entstandene populäre Wissen einen anderen Maßstab als den der wissenschaftlichen Rationalität anzulegen (Ebd.: 20-27).

Im Mittelpunkt neuerer Untersuchungen zu populärem Wissen steht insbesondere die Frage nach dem epistemischen Stellenwert von Bildlichkeit und Praktiken der Visualisierung (Nikolow and Bluma 2002; Nikolow and Bluma 2009; Heumann and Hüntelmann 2013: 283-293). Auch in Das Leben des Menschen wird deutlich, welche Rolle dem Sachbild bei der Zirkulation anatomischen und physiologischen Wissens zukam. Der Beitrag knüpft hier an, wobei nicht die mit der Bildlichkeit transferierten Wissensinhalte im Mittelpunkt stehen, sondern der Entstehungsprozess der Bilder, ihr Gebrauch sowie ihre Zirkulation. Hiermit eng verbunden ist der zweite Schwerpunkt meines Beitrags: eine Analyse des Produktions- und Publikationskontextes der Kahn'schen Illustrationen gibt Auskunft über die kollektive Struktur der Autorschaft eines der wichtigsten populärmedizinischen Werke der Zwischenkriegszeit. Anhand von Archivmaterial versuche ich mit diesem Beitrag 
die konkreten Produktionsprozesse der Bilder und damit die Zusammenarbeit zwischen Kahn, seinen mehr als 15 Illustratoren und der Franckh'schen Verlagshandlung zu rekonstruieren. Drei Zeichner werden näher anhand ihrer nachgelassenen Korrespondenz vorgestellt. Dies ermöglicht einen differenzierten Blick auf die unterschiedlichen Ebenen, in denen Autorschaft im Medium des populären Buches zum Ausdruck kommt. Ein stärkeres historisches Verständnis dieses Kollektivs, das sich im Herstellungsprozess der Illustrationen zeigt und das im Fleck'schen Sinne wohl auch als Denkkollektiv zu bezeichnen wäre (Fleck 1935/1980), führt nicht nur dazu, die stilistische Vielfalt im Kahn'schen Werk zu verstehen. Es eröffnet darüber hinaus - und dies ist eine zentrale These meines Beitrags - neue historische Perspektiven, um die vielfältige und durchaus kontroverse Rezeptionsgeschichte der Kahn'schen Illustrationen über den politischen Umbruch von der Weimarer Republik zum Nationalsozialismus einzuordnen.

Wegen Kahns jüdischer Abstammung wurde Das Leben des Menschen 1933 in Deutschland verboten. In der Franckh'schen Verlagshandlung erschien nun zunehmend populäre Literatur, die von nationalsozialistischer Propaganda durchdrungen war. Hierzu zählten Schriften, die der Arzt Gerhard Venzmer (1893-1986) in den 1930er Jahren bei Franckh herausgab, insbesondere seine Monographie Der Mensch und sein Leben (Venzmer 1938), in dem sich zahlreiche Anleihen bei Kahns Buchreihe finden, die nicht als solche ausgewiesen wurden. So stammen mehr als die Hälfte der Bilder in Der Mensch und sein Leben aus Fritz Kahns Das Leben des Menschen. Aufgrund dessen wurde Venzmers Buch schon von Fritz Kahn selbst als schlechte Kopie seiner eigenen Reihe bezeichnet (Kahn 1943a).

Aber nicht nur Venzmer griff auf die Abbildungen aus Das Leben des Menschen zurück; auch Kahn selbst verwendete die Bilder in seinen Exilwerken, die er in der Schweiz und den USA veröffentlichte. Die Illustrationen aus Das Leben des Menschen aus den 1920er Jahren konnten offenbar die Grundlage für weitere Veröffentlichungen in den 1930er Jahren bilden, die in unterschiedlichsten politischen und sozialen Kontexten erschienen. Die kollektive und vernetzte Produktion der Illustrationen zur Zeit der Weimarer Republik stellte, so meine These, den Ausgangspunkt für die konträren Weiterentwicklungen der populärmedizinischen visuellen Strategien dar, die sich in der ästhetischen und medialen Kontinuität dieser Wissensobjekte und der Übertragung des Kahn'schen Werkes sowohl in das US-amerikanische Exil als auch in den neu entstehenden Kanon der NS-Propagandaliteratur zeigen. Um diese Vielfalt der Verwendung und die damit verbundenen medialen Bedeutungsverschiebungen besser zu verstehen, entlehne ich für diesen Beitrag den Begriff ,Transkription', den der Linguist und Medienwissenschaftler Ludwig Jäger geprägt hat.

,Transkription“, zunächst verstanden als eine „Koppelung systemischer Texturen“ (Jäger 2004: 72), stellt „eine symbolische Operation wechselseitiger 
[...] Um-, Ein- und Überschreibungen dar, die als basale Strategie für die Generierung kultureller Semantik zu funktionieren scheint" (Jäger 2003: 3). Jägers Ansatz trägt zum Verständnis dazu bei, wie neue kulturelle Semantik unter Rückgriff auf ein vorhandenes Segment generiert wird, indem unterschiedliche Medien gekoppelt werden. Das ältere Segment verschwindet jedoch nicht, sondern tritt stattdessen in Beziehung und Wechselwirkung mit neuen Auffassungen, Meinungen und Verständnissen.

Aus dieser Perspektive sind sowohl Venzmers Der Mensch und sein Leben als auch Kahns Exilveröffentlichungen als Transkripte von Kahns Das Leben des Menschen zu lesen und der Prozess der Neuveröffentlichung ist als ein transkriptives Verfahren (Jäger 2004b: 341) zu verstehen. Kahn hatte mit Das Leben des Menschen ein Werk geschaffen, das sich (auch durch seine materielle Verfasstheit) als Prätext im Sinne Jägers auszeichnete und als Grundlage für neue Skripte diente (Jäger 2002: 30). ${ }^{1}$

Im ersten Teil des Beitrags stelle ich dar, wie sich Fritz Kahns geplante Veröffentlichung von Das Leben des Menschen in das Programm der Franckh'schen Verlagshandlung einfügte, einem Verlagshaus, das sich auf populäre Schriften spezialisiert hatte. Der zweite Teil hebt die Zusammenarbeit von Kahn und den Graphikern hervor, die Das Leben des Menschen illustrierten. Im dritten Teil widme ich mich der durch Gesetzgebungen verursachten Veränderung der verlegerischen Praxis des Verlagshauses Franckh im frühen Nationalsozialismus. In den Abschnitten vier und fünf zeige ich schließlich, wie Kahns Werk im Nationalsozialismus und im Exil neu veröffentlicht wurde.

\section{Fritz Kahns Kooperation mit der Franckh'schen Verlagshandlung (1912-1931)}

Seit dem ausgehenden 19. Jahrhundert haben sich einzelne Verlage auf die Herausgabe populärer medizinischer und naturwissenschaftlicher Darstellungen spezialisiert (Daum 2002: 185 f.). 1903 gründeten die Inhaber der Franckh'schen Verlagshandlung, Walther Keller und Euchar Nehmann, die "Gesellschaft der Naturfreunde", deren Mitglieder monatlich die Zeitschrift Kosmos - Handweiser für Naturfreunde erhielten und weitere Veröffentlichungen, wie die Kosmos-Bändchen, ${ }^{2}$ zum Subskriptionspreis erwerben konnten. Erklärtes Ziel des Verlags war es, „naturwissenschaftliche Information für jeden greifbar und leicht verständlich zugänglich zu machen“. ${ }^{3}$ Als qualifiziert für einen Werkvertrag erachteten die Inhaber des Verlagshauses Franckh ausgewiesene Fachleute auf einem naturwissenschaftlichen oder medizinischen Gebiet, die zugleich Kenntnisse über die populäre Darstellung von Wissenschaft vorweisen konnten. Walther Keller legte darüber hinaus 
besonderen Wert auf didaktische, klare Bilder, die im Verlag selbst als „Bilder zum Studieren “4 bekannt waren. Die Herstellung der Illustrationen des Kosmos sowie weiterer Veröffentlichungen lag in der Verantwortung von vier angestellten Verlagsgraphikern. ${ }^{5}$

Fritz Kahn, der bereits während seines Studiums ein ausgeprägtes Interesse an der populären Darstellung naturwissenschaftlichen Wissens gezeigt hatte (von Debschitz 2009: 25), unterzeichnete 1912 seinen ersten Werkvertrag. ${ }^{6}$ Zwei Paragraphen des Vertrags stechen besonders hervor: Der Abgabetermin und die Vereinbarung zur Illustrierung des Werkes. Als Abgabetermin des Buches, das im Jahr 1912 noch ein Werk über die „Biologie des Menschen“ werden sollte, wurde das Ende des Kalenderjahres 1913 festgelegt. Die Illustrationen sollte nicht ausschließlich von den hauseigenen Graphiker angefertigt werden, sondern es wurde vereinbart, dass Kahn selbst Vorschläge für das Bildmaterial liefern sollte. Ein kurz vor Beginn des Ersten Weltkrieges im Juni 1914 unterzeichneter Zusatzvertrag verdeutlicht, dass Kahn den Zeichnern nicht lediglich Bildideen vorschlug, sondern dass er selbst aktiv in die Bebilderung seines Buches einbezogen werden und „Vorlagen und Nachweise für das dazugehörige Abbildungsmaterial “7 liefern sollte. Zu diesem Zeitpunkt gab Kahn bei mindestens einem Zeichner Illustrationen in Auftrag, der nicht als Zeichner bei der Franckh'schen Verlagshandlung angestellt war. Der Maler Arthur Schmitson zeichnete für Kahn und wurde dafür von der Franckh'schen Verlagshandlung entlohnt. ${ }^{8}$ Allerdings wurden mit Ausbruch des Ersten Weltkrieges im August 1914 sowohl die Geschäftsbeziehung mit Schmitson als auch die Arbeit am Buch unterbrochen, da Kahn von 1914 bis 1918 Militärdienst als Sanitätsarzt leistete. Der Ablieferungstermin des Manuskripts wurde um die Dauer des Krieges verschoben. ${ }^{9}$

Nach dem Ersten Weltkrieg erschien 1919 - quasi als Vorläufer von Das Leben des Menschen - zunächst Die Zelle in der Reihe der Kosmos-Bändchen (Eilers 2012: 201). Fritz Kahn arbeitete zudem an einem weiteren Buch mit: Wunder in uns (Günther 1921) war ein Kompendium mit Beiträgen von acht Autoren, die naturwissenschaftliches und medizinisches Wissen popularisierten. Bereits im Titel wurde ein zeitgenössischer Topos aufgegriffen: ,Wunder' gehörten - mit im Laufe des 20. Jahrhunderts wechselnden Motiven - in das Genre des populären Wissens. Häufig wurden sie aus dem Bereich Natur und Technik rekrutiert und so passt Wunder in uns zur inflationären Darstellung von Wundern im populären Wissen: Das Wunder in uns verweist darauf, dass das Innenleben des Menschen zwar vertraut erschien, durch die Erkenntnisse der Medizin des 19. Jahrhunderts aber nicht vollständig erklärbar geworden war. Paradoxerweise wurden gerade solche Phänomene, die als nicht wissenschaftlich erklärbar galten, häufig zum Ausgangspunkt für popularisierende Darstellungen von Wissenschaft genommen. Diese Veröffentlichungen zeichneten sich „weniger durch Materialismus, Positivismus [...] als durch Idealismus, Einheitsgedanken, Ästhetisierung und 
Wiederverzauberung“ aus (Gall 2011: 287). Das Motiv der Wiederverzauberung führte bei Kahn allerdings - im Gegensatz zu anderen populären Darstellungen - weder zu einer religiösen noch zu einer holistischen Deutung der Welt. Vielmehr war ein häufiger Gebrauch von Technikmetaphern zu verzeichnen und hierbei insbesondere die Koppelung von menschlicher Biologie und technischer Analogie. Hierbei handelte es sich um ein Wahrnehmungsmuster, das Ausdruck der zeitgenössischen Technikbegeisterung war (ebd.: 276). In Wunder in uns veröffentlichte Kahn drei Texte. Die Erstauflage erschien 1921 und war so schnell vergriffen, dass bereits ein Jahr später eine bearbeitete Neuausgabe in Druck ging.

Die stärkste Veränderung im Vergleich zur ersten Auflage waren acht kolorierte Tafeln, deren Design einen für den deutschen Buchmarkt neuartigen Visualisierungsstil zeigte. Die Anordnung von Text, Illustration und Fotografie hatte der Herausgeber Hanns Günther dem Stil amerikanischer Zeitungen und Magazine entlehnt; allen voran der US-amerikanischen Bildenzyklopädie Pictured Knowledge (Sappol 2012: 160). Wunder in uns führte eine amerikanische Methode in den deutschen Kontext von Popularisierung ein: Autoren und Illustratoren verknüpften erstmals Körperfunktionen mit der industrialisierten Lebenswelt der USA (ebd.). Zahlreiche Tafeln weisen Ähnlichkeiten mit den Visualisierungsstrategien auf, die Kahn später berühmt machen sollten: Der schematische Torso in Seitenansicht, dessen Blick sich nach links wendet, dessen Innenleben von Homunkuli bevölkert ist und in dem Pfeile die Bewegung der Lebenssäfte anzeigen. Die Figur, die die Grundstruktur in Der Mensch als Industrieplast liefert, findet sich bereits hier in Grundzügen.

Der Werkvertrag, der Illustrationen für die Bücher aus der Franckh'schen Verlagshandlung explizit vorsah, und die kolorierten Tafeln in Wunder in uns sind Indizien für einen sich wandelnden Umgang mit Bildern in populärer naturwissenschaftlicher und medizinischer Literatur. So hatte beispielsweise Wilhelm Bölsche, im frühen 20. Jahrhundert einer der bekanntesten Autoren, die naturwissenschaftliches Wissen einer breiten Öffentlichkeit zugänglich machten, noch 1913 eine kritische Distanz zu Bildern gefordert. In seinem Aufsatz „Wie und warum soll man die Naturwissenschaft ins Volke tragen?" führte er aus: „Aber im populären Buch meine ich, daß eine wirklich gute Rede vielfältig mehr wert ist als noch so viel Bilder" (Bölsche 1913: 20). Dessen ungeachtet erkannte Bölsche die zunehmende Bedeutung von Illustrationen und stellte Überlegungen dazu an, welche Qualitäten diese Bilder aufweisen sollten. Bilder in populären Werken sollten als eigenständige Werke produziert und nicht unverändert aus dem akademischen Betrieb übernommen werden: „auch die fachwissenschaftlichen Abbildungen müssen für den volksbelehrenden Zweck durchweg noch einmal ,umgesehen', noch einmal umgezeichnet werden. Wo dem Fachmann das Bild alles ihm wichtige zeigt, da zeigt es dem Laien in einer erdrückenden Masse von Fällen überhaupt noch nichts" (ebd.). 
Innerhalb von zehn Jahren nach Bölsches Feststellung nahm die Verwendung von Bildern zur Illustration populären Wissens in qualitativer und quantitativer Hinsicht zu. Bestanden Bölsches Werke fast ausschließlich aus Text, blieb in Kahns Büchern kaum eine Seite ohne Bild. Der Forderung nach eigenständiger Bildproduktion für dieses Genre wurde dabei mehrfach nachgekommen: Zum einen arbeitete Fritz Kahn für ein Verlagshaus, das eigens für den Zweck der Illustration populärer Werke Zeichner angestellt hatte. Zum anderen kooperierte Kahn selbst mit Zeichnern, die professionelle Werbezeichner oder anatomische Illustratoren waren und anatomische und physiologische Zusammenhänge, wie Bölsche es gefordert hatte „umsahen“. Nicht zuletzt hatte er durch seine Beiträge in Wunder in uns die Analogie von industriellen und physiologischen Prozessen bei der Darstellung des Körpers kennengelernt.

1922, als die zweite Auflage von Wunder in uns erschien, begann die Franckh'sche Verlagshandlung mit der Herausgabe von Kahns Buch zur Biologie des Menschen, das inzwischen zu einer ganzen Buchreihe herangewachsen war. Nicht mehr wie zunächst geplant 21, sondern 64 Druckbogen ${ }^{10}$ und somit fünf Bände umfasste das Gesamtwerk, als im Jahr 1931 der letzte Teil veröffentlicht wurde. Inzwischen hatte es den lehrbuchartigen Titel „Biologie des Menschen“ gegen einen Titel eingetauscht, der sich bestens in den bestehenden Kanon populären Wissens im Kosmos-Verlag einfügte: Nach Bölsches Das Liebesleben in der Natur in drei Bänden (Bölsche 1898-1903) und Raoul Heinrich Francés Das Leben der Pflanze (Francé 1921) erschien nun Fritz Kahns Das Leben des Menschen. Die letzten Veröffentlichungsmodalitäten für das Werk wurden 1921 durch einen weiteren Nachvertrag geklärt. Als allein verantwortlich für die Illustrationen zeichnete Kahn. Er würde fortan „alle nötigen Angaben und Vorschläge für das Bildmaterial liefern und die Zeichner entsprechend überwachen". ${ }^{11}$ Weiterhin wurde vereinbart, dass gegen Zahlung eines Honorars die ausschließlichen Verlags- und Urheberrechte in den alleinigen Besitz der Franckh'schen Verlagshandlung übergingen. $^{12}$

Die Bücher fanden reißenden Absatz, wobei Leser und Presse den Text als spannend und leicht verständlich sowie die Bilder als plastische und greifbare Darstellung des Menschen rezipierten. Das Leben des Menschen dürfte zu den am häufigsten verkauften Büchern aus dem Hause Franckh gehört haben und zählte in der Deutschen Bibliothek Leipzig zu den meistverlangten Werken. ${ }^{13}$ Die Leserbriefe an Kahn zeigen, dass die Bücher in allen sozialen Schichten gelesen und von medizinischen Laien, von wissenschaftlicher ebenso wie von esoterischer Seite rezipiert wurden. ${ }^{14}$ Neben vielen anderen Medizinern gehörte der Arzt und Schriftsteller Alfred Döblin zu den Rezensenten, die das Werk aufgrund seines eingängigen Stils und der neuartigen Illustrationen besonders empfahlen: „Das Buch muß aus allen vorliegenden ähnlichen volkstümlichen Werken besonders hervorgehoben werden, wegen der 
Leichtigkeit und Originalität des Vortrags, besonders wegen der Originalität und Schlagkraft der bildlichen Darstellung" (Döblin 1922: 1231 f.). Andere Rezensenten wiesen noch stärker auf die enge Verzahnung von Bildmotiv und urbaner Lebenswelt hin, zu der die Industrialisierung ebenso wie die Reklame gehörte. So urteilte etwa die Buchbesprechung der Vossischen Zeitung, einer überregionalen Tageszeitung aus Berlin:

Das Charakteristische der Kahnschen Wirkung möchte besonders hier zu finden sein: er überträgt die Effekte der modernen Großstadt-Reklamen auf den wissenschaftlichen Unterricht. Manche dieser Bilder, die das Maschinenhafte des Organischen betonen, drängen sich der Phantasie mit der Hartnäckigkeit von Halluzinationen auf. ${ }^{15}$

Auch alle weiteren Rezensenten nannten Kahn als einzigen Autor der Buchreihe und schrieben ihm desgleichen alle Illustrationen zu. Diese Sichtweise hat sich bis heute gehalten. Bereits ein erster Blick in die Bücher macht jedoch unmittelbar klar: Die Bilder wurden nicht von Kahn selbst angefertigt. Viele Bilder waren als Fotografien aus dem Kosmos-Handweiser übernommen, es gab nicht ausgewiesene Fotografien sowie Replikationen bekannter Kunstwerke, etwa von da Vinci, Michelangelo, Lenbach oder Rodin, die einen hohen Wiedererkennungswert bei Kahns bildungsbürgerlichem Publikum aufgewiesen haben dürften.

Insbesondere jene Bilder, denen die Leser Attribute wie „märchenhaft" oder „der Großstadtwerbung nachempfunden“ beigaben, waren aus einer Zusammenarbeit zwischen Kahn und seinen Illustratoren hervorgegangen. Es ist zu vermuten, dass alle Illustratoren in Berlin wohnten und in persönlichem Kontakt mit Fritz Kahn standen, der die Bilder bei ihnen in Auftrag gab. Für die meisten Illustrationen lassen sich die Zeichner durch ihre Signaturen identifizieren und die Ordnung der Bilder nach ihren Illustratoren lässt verschiedene Stile erkennen. Insgesamt lassen sich mindestens 15 Zeichner ausmachen, die exklusiv für Kahn arbeiteten und in keinem Arbeitsverhältnis mit der Franckh'schen Verlagshandlung standen.

Im Verlauf der jahrelangen Arbeit an Das Leben des Menschen war die Kooperation mit den einzelnen Zeichnern unterschiedlich stark ausgeprägt und abhängig von den Themen, die Fritz Kahn in seinen Büchern darlegte. Bei der Auswahl seiner Illustratoren legte er großen Wert auf deren Spezialisierung als professionelle Zeichner. Angestellte Verlagszeichner seien seiner Meinung nach nicht in der Lage, wissenschaftliche Zeichnungen anzufertigen. ${ }^{16}$ Die Produktion von Bildern in populären Veröffentlichungen entwickelte sich als eigenständiges Genre: Nicht unter dem Mikroskop oder im Röntgenlabor, sondern in Kahns Schreibstube, im Atelier oder Zeichenbüro wurden Bilder als kommerzielles Gut entworfen und hergestellt. Dabei sollte das Bild hinsichtlich seiner Relevanz und der (historisch so definierten) Exaktheit seiner Darstellungen wissenschaftlichen Kriterien entsprechen. Im Folgenden werden die Arbeiten von drei Graphikern 
vorgestellt, deren Briefwechsel mit der Franckh'schen Verlagshandlung erhalten geblieben ist: Arthur Schmitson, Fritz Schüler und Roman Rechn.

\section{Bilder herstellen - Die Zeichner Arthur Schmitson, Fritz Schüler und Roman Rechn}

In den ersten beiden Bänden steuerte Arthur Schmitson (geboren wahrscheinlich 1857, Todesjahr unbekannt) mehr als dreißig Bilder bei, darunter viele ganzseitige Bildtafeln. Schmitsons Bilder stehen in der Tradition der anatomischen Atlanten. Als Bildzitate finden sich Illustrationen aus dem Atlas des französischen Anatomen Jean Marc Bourgéry (1797-1849), der an seinem Traité complet de l'anatomie über zwanzig Jahre hinweg mit dem Zeichner Nicolas Henri Jacob arbeitete. Arthur Schmitson wirkte vor der Zusammenarbeit mit Kahn am ersten und zweiten Band der Anatomieatlanten von Johannes Sobotta mit, ein Atlas für Medizinstudenten und Ärzte, der bis heute neu aufgelegt wird. Im Vorwort des ersten Bandes erwähnte Sobotta seine Zeichner Carl Hajek (1878-1935) und Arthur Schmitson. Die Zusammenarbeit mit Schmitson beendete Sobotta zu Gunsten von Hajek, der den zweiten und dritten Band von Sobottas Atlas hauptsächlich illustrierte (Sobotta 1904: VII). Nach der Zusammenarbeit mit Sobotta brachten Hajek und Schmitson ihre anatomischen Zeichnungen in unterschiedliche Kontexte ein: Während Hajek für den Wiener Anatomen und sozialdemokratischen Gesundheitsreformer Julius Tandler (1869-1936) arbeitete (vgl. Nemec 2015) und hier zu einem medizinischen Reformprojekt beitrug, das den ,neuen Menschen“ formen sollte (ebd.), ${ }^{17}$ begann Schmitson mit der Illustrierung von Kahns Buchreihe. Das Leben des Menschen war mit seinen anatomischen Abbildungen versehen, die durch ihre naturalistische Genauigkeit bestachen, wie die Darstellung des Herzens zeigt (Abb. 2).

Die Bildzitate aus dem Bougéry können genau wie die anatomischen Darstellungen von Schmitson in Zusammenhang mit wissenschaftlicher Objektivität gebracht werden, das heißt mit der Frage, wie die Natur exakt abzubilden sei. Auf die historische Kontingenz der Antwort auf diese Frage haben Lorraine Daston und Peter Galison mit ihrem Begriff der „mechanischen Objektivität" hingewiesen, den sie unter anderem an dem Sobotta Atlas geschärft haben (Daston and Galison 2007: 175-177). Die Autoren stellen heraus, dass Sobotta für seinen Atlas, im Gegensatz zu anderen zeitgenössischen Werken, neue Verfahren der Bildherstellung wählte. Er verzichtete auf Holzschnitte, da diese dem Verfasser nicht naturgetreu genug waren und der Interpretation des Künstlers zu viel Spielraum ließen (Daston and Galison 2007: 367). Wie Daston und Galison zeigen, hatte Sobotta sich dafür entschieden, Objektivität durch ein Verfahren herzustellen, dessen 


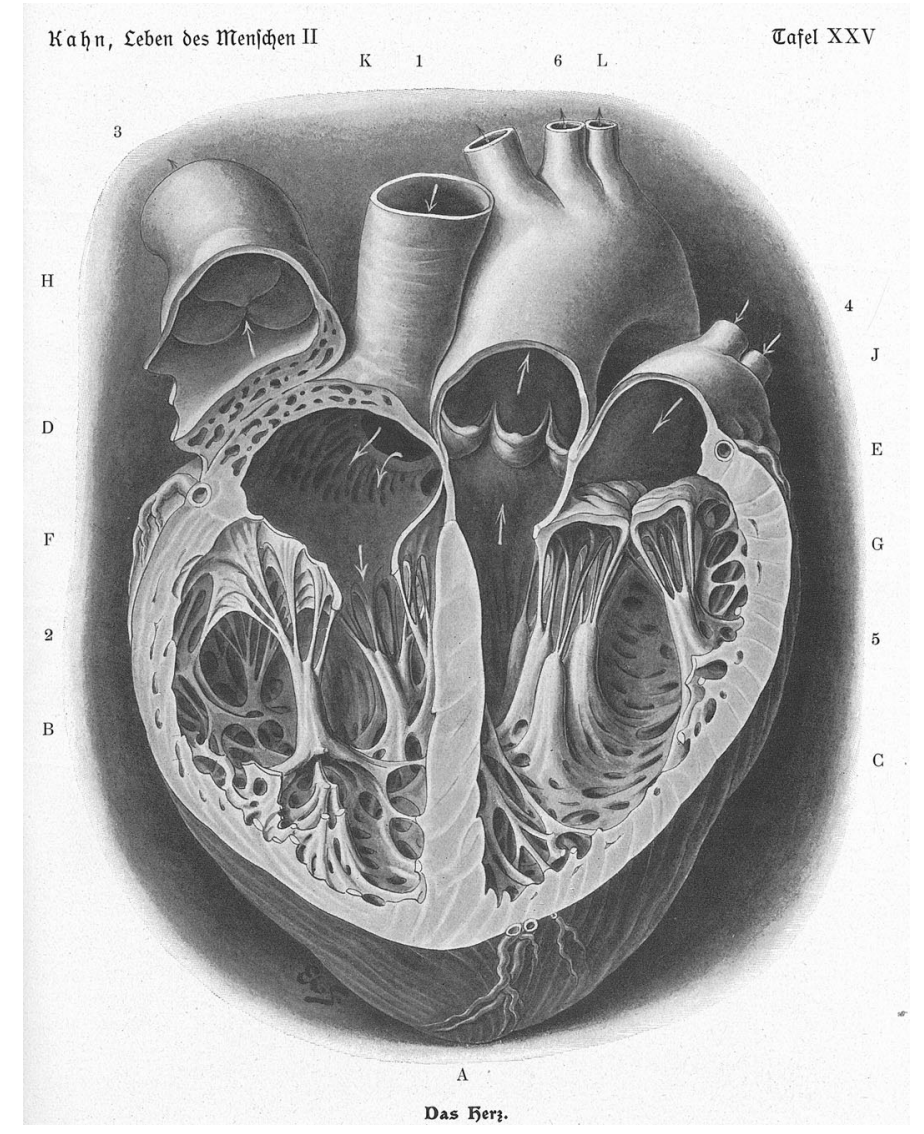

Abb. 2 "Das Herz" (Quelle Das Leben des Menschen. Band 2. 1924. Copyright FranckhKosmos, Stuttgart)

Ausgangspunkt mehrere Fotografien waren und auf dessen Basis dann Zeichnungen entstanden (ebd.: 175). Für Daston und Galison ist der Atlas ein Beispiel dafür, wie im späten 19. und frühen 20. Jahrhundert neu verhandelt wurde, was als naturgetreue und damit objektive Darstellung gelten durfte.

Die anatomischen Atlasbilder weisen darauf hin, dass auch im Diskurs des öffentlichen Wissens die Frage nach Objektivität eine Rolle spielte - wenn auch unklar bleibt, welche theoretische Vorstellung von Objektivität Kahn hatte und als Maßstab vorgab. Schmitsons Stil steht für eine Form von Objektivität, die als Bildzitat und damit als Referenz auf akademische Werke diente. Zugleich aber stehen seine Arbeiten für eine freiere Interpretation, die mit dem Stil der mechanischen Objektivität brach: Bezieht man das gesamte Oeuvre ein, das Schmitson für Kahn anfertigte, ist am Beispiel der Illustration Im Talgrund einer Fleischwunde (Abb. 3) die Vermischung von naturalistischmikroanatomischer Darstellung mit phantastischen Elementen $\mathrm{zu}$ verzeichnen. 


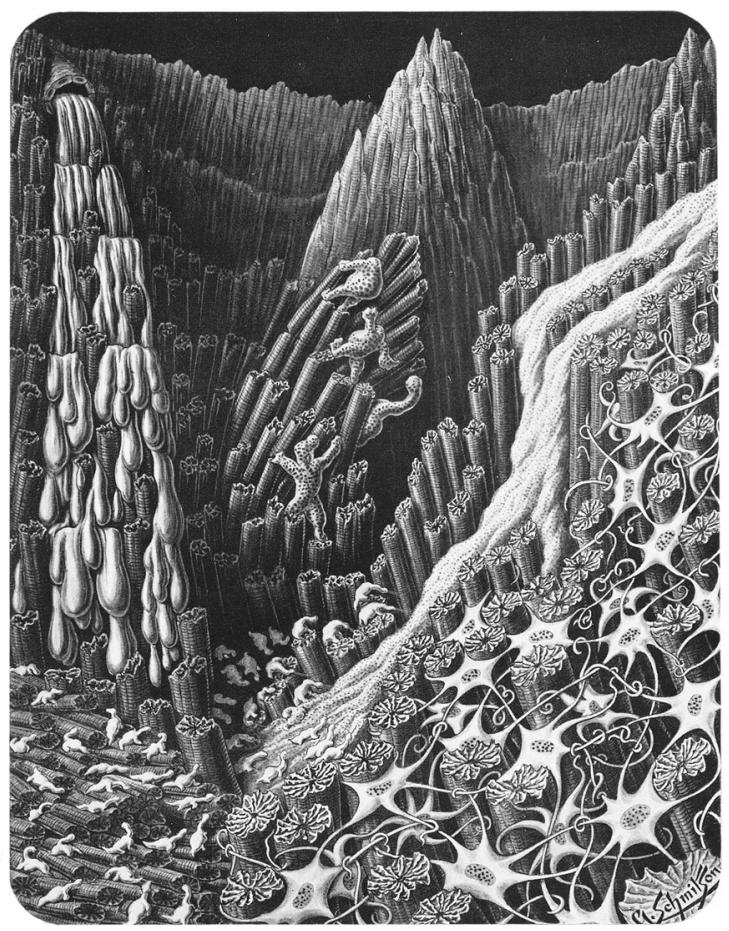

Reifeerlebniffe einer खa anderz̨elle I.

Abb. 3 "Reiseerlebnisse einer Wanderzelle I" (Quelle: Das Leben des Menschen. Band 2. 1924. Copyright Franckh-Kosmos, Stuttgart)

Kahns Kurzgeschichte Meine Erlebnisse als Wanderzelle schloss den zweiten Band von Das Leben des Menschen (1924) ab. Schmitson illustrierte diese Erzählung mit einer Bildserie aus vier Tafeln, die den Titel Reiseerlebnisse einer Wanderzelle tragen (vgl. Abb. 3). Der Wandel von der anatomischen Atlaszeichnung hin zu einer künstlerischen Bildgeschichte und damit das Nebeneinander von „Idealen der Naturweisheit und der mechanischen Objektivität" (Daston and Galison 2007: 261) deutet die eigenständige Darstellung des menschlichen Körpers und seiner Funktionen im Genre des populären Wissens an. Diese Darstellungsweise löste sich von den Illustrationen aus fachmedizinischem Kontext und installierte ein eigenes Bild- und Bildungsprogramm. Als dies populärer wurde, verschob sich die Zusammenarbeit zwischen Kahn und den Illustratoren vom anatomischen Zeichner hin zum Architekten, Ingenieur und Werbegraphiker, deren Stil sich in Wunder in uns bereits angedeutet, aber noch nicht etabliert hatte ${ }^{18}$. Dieser Stil folgte nicht notwendigerweise den Prämissen mechanischer Objektivität, sondern erlaubte Darstellungen des Menschen, die auf didaktische Verständlichkeit statt auf anatomische oder physiologische Genauigkeit setzten. 
Mit der Arbeit am dritten Band, der 1926 erschien, begann Fritz Kahns Kooperation mit weiteren Zeichnern, unter ihnen Roman Rechn und Fritz Schüler. Diese Zeichner griffen nun eine künstlerische Linie auf, die aus Wunder in uns bekannt war. Hatten in den ersten beiden Bänden von Das Leben des Menschen Darstellungen vorgeherrscht, die in der Tradition des anatomischen Realismus oder des Jugendstil standen, hielten nun immer mehr Illustrationen Einzug, die den Menschen in seiner technischen Lebenswelt zeigten und - umgekehrt - die neue technisch-funktionale Lebenswelt der 1920er Jahre im Menschen widerspiegelten. Die von Roman Rechn (1892-1945) angefertigten Bilder finden sich im vierten und fünften Band. Seine spärlichen biographischen Daten verweisen auf einen beruflichen Werdegang als Zeichner: Geboren in Estland studierte er in Riga Architektur. Seit 1920 lebte er in Berlin und arbeitete hauptberuflich bei großen Berliner Unternehmen als Graphiker. Bis 1922 war er Angestellter im technischen Büro der Siemens-Schuckert Werke und gleichzeitig als freiberuflicher Graphiker und Kunstgewerbler tätig. Danach wechselte er als Graphiker zum Buchverlag Ullstein in Berlin, wo er bis März 1923 blieb. Im Jahr 1924, nachdem er sich dem Kunstgewerbe zugewandt hatte, bewarb er sich bei Walter Gropius um die Aufnahme zum Studium am Bauhaus in Weimar, die jedoch von Gropius, Paul Klee und Josef Hartwig abschlägig beschieden wurde. ${ }^{19}$ Roman Rechn illustrierte makro- und mikroskopische Anatomie und seine Zeichnungen lösten zunehmend die Illustrationen von Arthur Schmitson ab.

Rechn situierte Anatomie und Physiologie in alltäglichen Handlungen (Abb. 4): In Rechns Bild, das auch das Umschlagbild des dritten Bandes von Das Leben des Menschen war, werden zeitgenössische mikroanatomische Darstellungen, biochemische Schematisierungen von Stoffwechsel und allägliche Praktiken wie beispielsweise der Vorgang des Trinkens montiert, wodurch die Grenze zwischen Naturwissenschaft und Alltagspraxis verschwimmt. Fritz Schüler (Lebensdaten unbekannt) war Ingenieur und schuf das Poster „Der Mensch als Industriepalast“, das 1926 als Beilage des vierten Bandes von Das Leben des Menschen veröffentlicht wurde. Er kombinierte die technische Ingenieurzeichnung mit „kommerziellem Realismus“ (Sappol 2013), das heißt mit einer bildlichen Wiedergabe, die charakteristisch für die Werbung in den Illustrierten der 1920er Jahre war.

Vier wesentliche Stilmerkmale in Das Leben des Menschen, die einzelnen Zeichnern eindeutig zuzuordnen sind, sind anatomischer Realismus (1, Schmitson) und Elemente des Jugendstils (2, Schmitson), die Übertragung alltäglicher Motive auf medizinische Aufklärung (3, Rechn) sowie die Erklärung von Physiologie durch technische Analogien (4, Schüler). Sie sind eine Erklärung für die stilistische Inkonstanz innerhalb des Gesamtwerkes. Innerhalb der einzelnen Bände jedoch, die maßgeblich von jeweils einem Illustrator geprägt wurden, entsteht der Eindruck konsistenter Visualisierungsstrategien. 


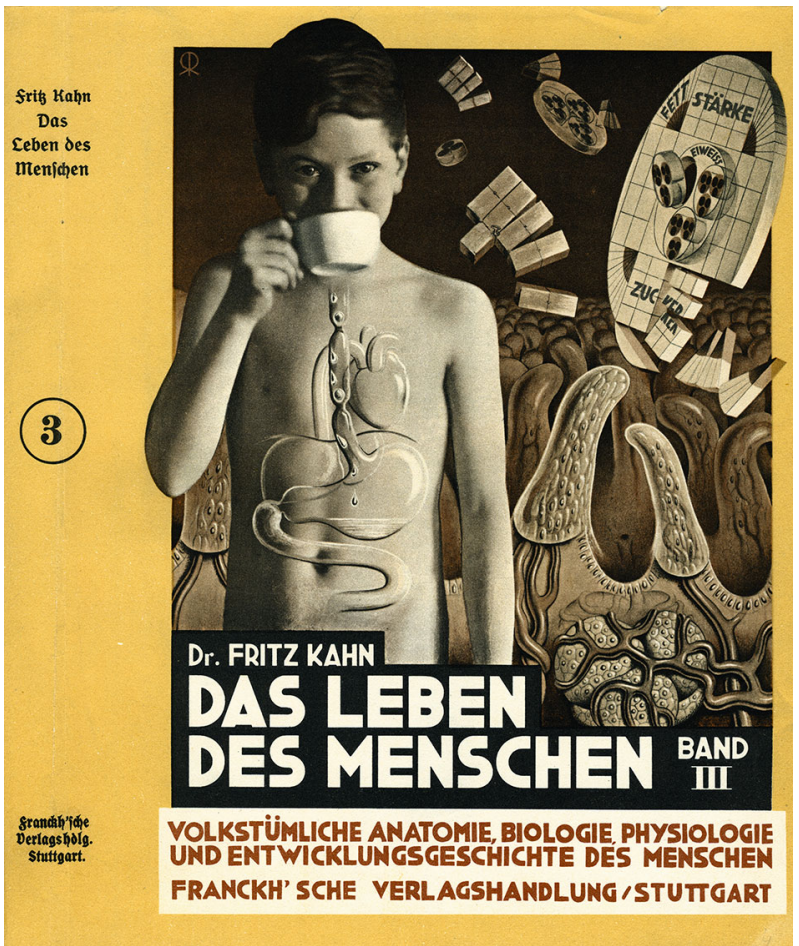

Abb. 4 Schutzumschlag „Das Leben des Menschen“ (Quelle: Das Leben des Menschen. Band 3. 1926. Copyright Franckh-Kosmos, Stuttgart)

Wie arbeiteten Kahn und seine Zeichner zusammen, was haben sie besprochen und wie oft gingen Entwürfe zwischen Kahn und seinen Zeichnern hin und her, bis die Illustration fertig war? Um diese Fragen beantworten zu können, wäre die Analyse des Originalmanuskripts oder der einzelnen Bildentwürfe notwendig. Das Originalmanuskript von Das Leben des Menschen ist jedoch, ebenso wie die meisten Bildentwürfe, im Zweiten Weltkrieg verloren gegangen. Allerdings lassen sich aus anderen nachgelassenen Manuskripten, die in direkter zeitlicher Nähe zu Das Leben des Menschen entstanden sind, Vermutungen darüber anstellen, wie sich der Produktionsprozess der Kahn'schen Bücher dargestellt haben könnte. Ab 1930 arbeitete Kahn an seinem unveröffentlicht gebliebenen Werk Die Naturgeschichte Palästinas, das in mehreren Entwürfen unterschiedlichen Datums vorliegt. ${ }^{20}$ Neben dem gebundenen, illustrierten Manuskript finden sich im Nachlass Skizzenbücher sowie bearbeitete Skizzen von Kahn und einem unbekannten Zeichner. Die einzelnen Seiten des Manuskripts weisen mehrere Schichten unterschiedlicher Materialien auf. Die unterste Schicht besteht aus einem dicken Grundpapier, auf das dünnere Papierschnipsel aufgeklebt sind, darunter Textelemente, aus einem Zeichenbogen ausgeschnittene und 
eingeklebte Skizzen eines Zeichners sowie eingerahmte Blattsegmente mit dem Vermerk „sketch“ als Platzhalter für eine Skizze, die noch in Planung oder zur Produktion beim Illustrator war.

Diese Zeichnungen haben eine eigene, sequenzielle Geschichte, die im Folgenden kurz nachvollzogen wird. Wie in Das Leben des Menschen plante Fritz Kahn in Die Naturgeschichte Palästinas die Erklärung komplizierter Naturphänomene durch alltägliche Beispiele. Die Zeichnungen zur Erklärung von Trocken- und Regenzeit beginnen mit einem von Kahn angefertigten, groben Entwurf (Abb. 5). Seine losen Planungsbögen aus Pergamentpapier weisen Symbole für die Elemente auf, die an der Entstehung von Regen- und Trockenzeit in Palästina beteiligt sind: Azoren und persischen Golf, als verbindendes Ikon einen Regenschirm; der noch ungeschriebene Erklärungstext ist nur durch wenige Linien angedeutet.

In einer zweiten Bildversion (Abb. 6), die sich in einem gebundenen Skizzenheft befindet, sind diese Symbole zu einem Bild zusammengefügt. In dieses Bild haben Kahn und eine weitere Person Bilderklärungen eingefügt. Die

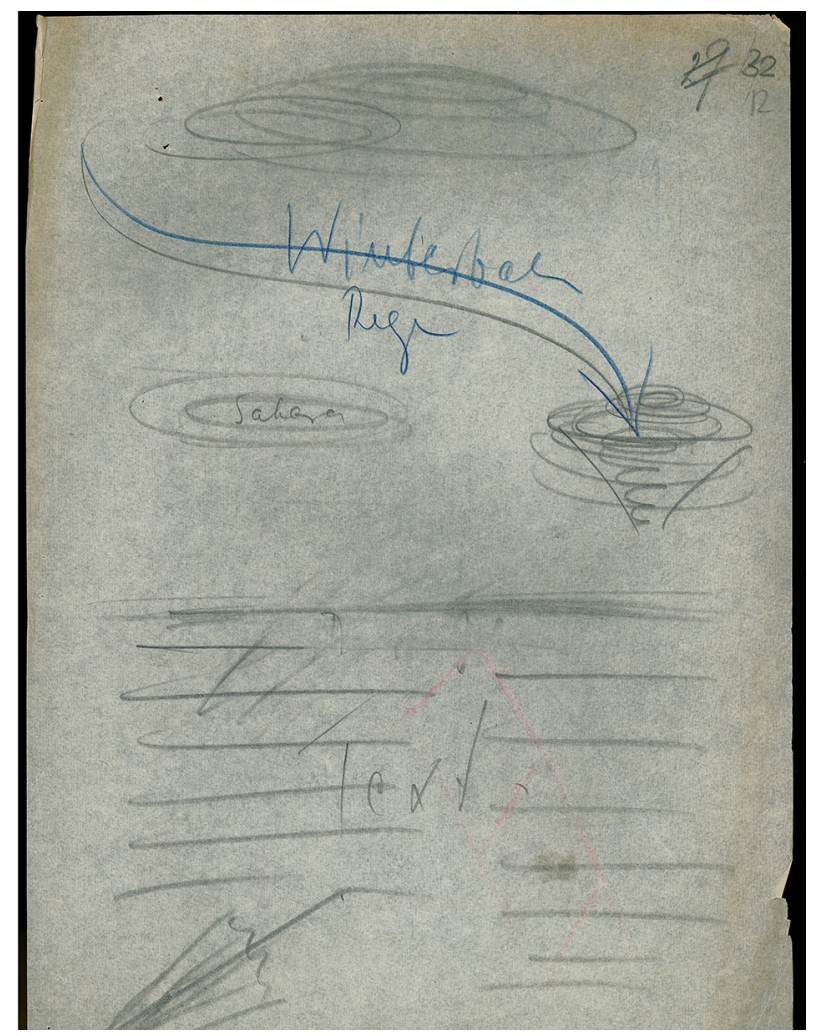

Abb. 5 Manuskriptskizze aus "Die Naturgeschichte Palästinas" (Quelle: Arthur and Fritz Kahn Collection; AR 7144/MF 746; Box 3; Folder 23; Courtesy of the Leo Baeck Institute) 


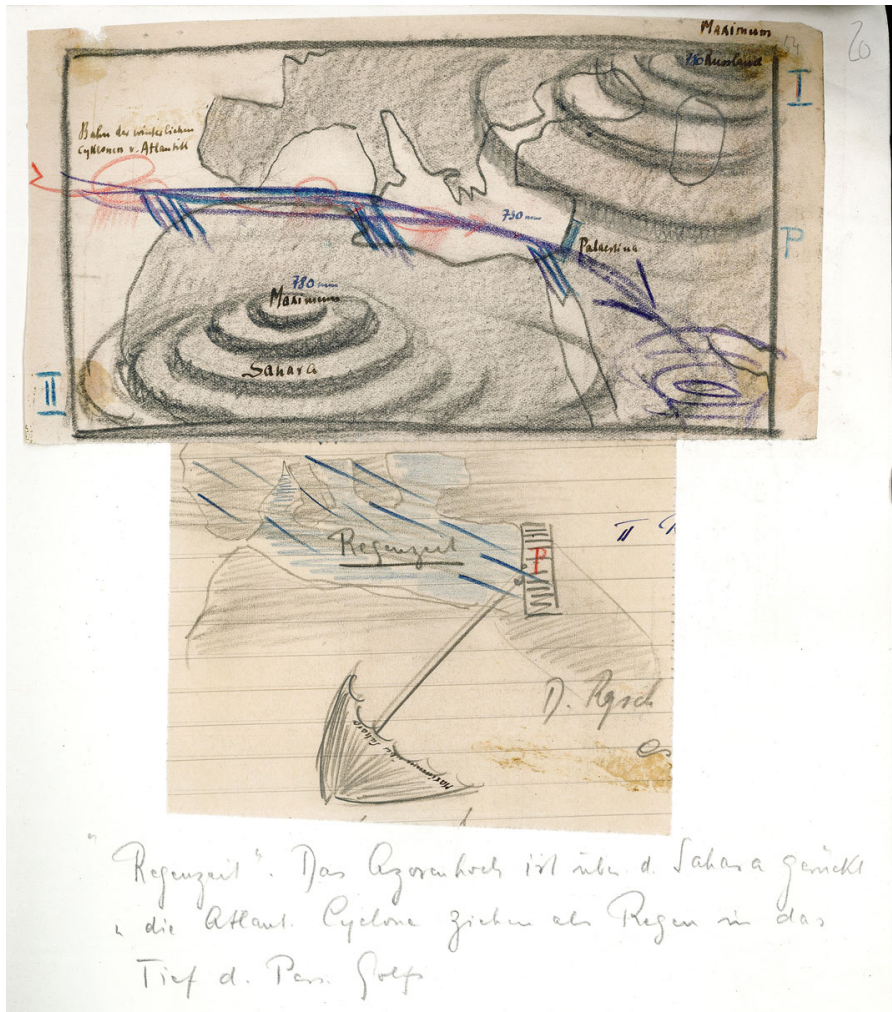

Abb. 6 Manuskriptskizze aus „Die Naturgeschichte Palästinas” (Quelle: Arthur and Fritz Kahn Collection; AR 7144/MF 746; Box 3; Folder 23; Courtesy of the Leo Baeck Institute)

Bildunterschrift hat Fritz Kahn selbst handschriftlich ausformuliert. Der dritte Teil der Sequenz (Abb. 7) besteht schließlich aus der fertigen Illustration, die Kahn ausgeschnitten und in sein Manuskript eingeklebt hat. Das Bild ist im Vergleich zu der ersten Planungsskizze hinsichtlich seiner visuellen Qualität ausgefeilter und offensichtlich von einem Illustrator bearbeitet worden. Kahn klebte diese Illustration auf das Grundpapier seines Manuskripts, auf dem er bereits die Bildunterschrift notiert hatte. Eine andere Bildsequenz zur Erklärung der Entstehung des Regens zeigt, dass Kahn in seine erste Skizze Anweisungen für den Illustrator schrieb. Als visuelle Vorlage diente eine Zeichnung, die Kahn aus einem dritten Werk ausgeschnitten hatte und neben der er vermerkte: „Zeichnung soll in der Art dieser Vorlage ausgeführt werden, diese Vorlage gehört nicht zur Ill“ (Abb. 8). ${ }^{21}$

Durch die im Archiv dokumentierte Produktion der Naturgeschichte Palästinas lassen sich in der Zusammenschau mit den signierten Illustrationen Rückschlüsse auf die Produktion von Das Leben des Menschen ziehen. Das Material zeigt, dass durch die Differenzierung des Produktionsprozesses in 


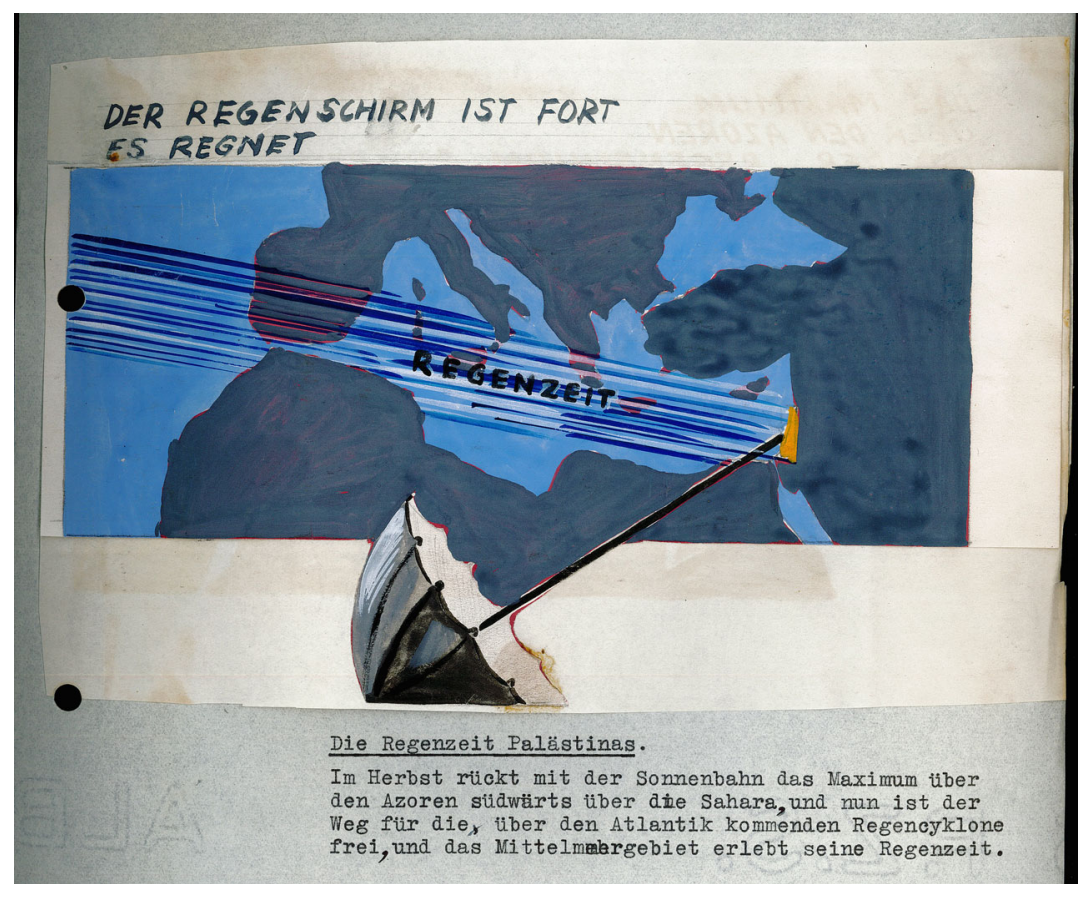

Abb. 7 Manuskriptskizze aus „Die Naturgeschichte Palästinas” (Quelle: Arthur and Fritz Kahn Collection; AR 7144/MF 746; Box 3; Folder 1; Courtesy of the Leo Baeck Institute)

Planung und Ausführung, in Thema und Idee, die sich bis zur Abgabe des Manuskripts an den Verlag mehrfach wiederholt haben wird, noch niemand auszumachen war, dem das Bild allein gehörte. An den Skizzen lässt sich ablesen, dass Kahn sich die Bilder ausdachte und seine Ideen in Bilder umsetzen ließ. Dabei hatte er genaue Vorstellungen, was die Illustrationen zeigen sollten. Unter seiner „Anleitung“, wie es in einem Brief von Franckh an Kahn 1922 formuliert wurde, lernten sie zu sehen und zu zeichnen. ${ }^{22}$

Der Titel der fertigen Buchreihe wurde unter Fritz Kahns Namen geführt, so dass die Bilder mit ihm allein in Verbindung gebracht werden konnten. Über den darstellenden Haupttext hinaus bestand die Rolle von Fritz Kahn fortan darin, eine festgelegte Erscheinungsweise visueller Aufklärungskultur zu charakterisieren. Sie bestimmte die Zirkulation und die Funktion dieser Art des gesundheitsaufklärerischen Diskurses in der Gesellschaft der Weimarer Republik. Auf den Bildern war dennoch ein Verweis auf das ungenannt gebliebene Zeichnerkollektiv zu finden: Die Graphiker signierten ihre Illustrationen und ihre Signatur blieb als Rückverweis auf den Produktionsprozess erhalten, der nach Fertigstellung der Buchreihe insofern anonymisiert worden war, als dass die Illustrationen allein Kahn in der öffentlichen Rezeption zugeschrieben wurden. Im Jahr 1931 waren alle fünf Bände von Das Leben des Menschen erschienen. Die Illustrationen der Buchreihe führten inzwischen ein 


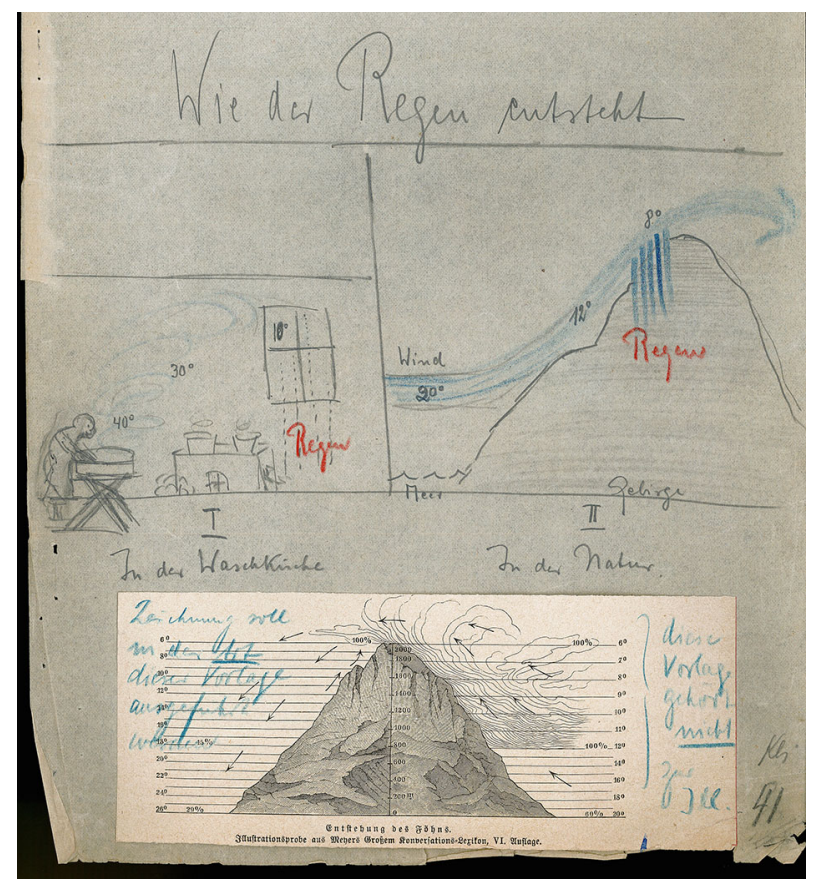

Abb. 8 Manuskriptskizze aus „Die Naturgeschichte Palästinas“ (Quelle: Arthur and Fritz Kahn Collection; AR 7144/MF 746; Box 3; Folder 1; Courtesy of the Leo Baeck Institute)

erstaunliches, von Das Leben des Menschen. Als Bildzitate nutzte Kahn selbst die Zeichnungen aus seinen Büchern, um damit Artikel für nationale und internationale Tageszeitungen und Magazine zu illustrieren. ${ }^{23}$ Franckh nutzte den Erfolg der Bücher ebenfalls und verwendete die Bilder im KosmosHandweiser, dem Publikationsorgan der Gesellschaft der Naturfreunde. ${ }^{24}$ Die Bilder wurden unter Angabe der Quelle in den Kosmos-Beiträgen anderer Autoren verwendet - insbesondere auch von Gerhard Venzmer, wie weiter unten noch detailliert dargestellt wird (Venzmer 1930a: 41-47).

Vor dem Erscheinen des letzten Bandes der Reihe Das Leben des Menschen bekundete der Verlag Franckh sein Interesse an den Zeichnungen. Im Mai 1931 sandte der Verlag den an der Publikation beteiligten Illustratoren so genannte „Verpflichtungsscheine ${ }^{\text {“ }}{ }^{25}$ mit deren Unterzeichnung sie sich verpflichten sollten, die Bilder, die sie für Kahn angefertigt hatten, weder Dritten anzubieten noch $\mathrm{zu}$ veräußern. Während Roman Rechn die Erklärung unterschrieb, ${ }^{26}$ verweigerte Fritz Schüler dies und behielt sich den Verkauf seiner Illustrationen vor. ${ }^{27}$ Mit dem Schlussband würdigten die Inhaber des Verlagshauses in einem Brief an Kahn die Gesamtpublikation als ein Ereignis mit dem „die Arbeit an ihrem schönen Lebenswerk vollendet scheint, soweit man dabei überhaupt von einem Ende sprechen kann “ ${ }^{28}$ Sie erinnerten auch an den Beginn der Zusammenarbeit im Jahr 1912, einer Zeit, die beinahe 
mythisch sei und in einer Zeit läge, ,die uns jetzt unglaublich ruhig und sicher erscheint". ${ }^{29}$ Nur zwei Jahre später nahm Kahns Leben eine abrupte Wende; Ende 1933 floh Kahn mit seiner Familie nach Palästina und seine Bücher wurden verboten.

\section{Franckh und Kahn: Veränderung verlegerischer Praxis 1931-1939}

Bei der von den Nationalsozialisten am 10. Mai 1933 inszenierten „Aktion wider den undeutschen Geist" wurden die Bücher jüdischer, marxistischer, pazifistischer und anderer oppositioneller oder politisch unliebsamer Autorinnen und Autoren verbrannt - darunter auch Das Leben des Menschen. Im September 1933 folgte die Gründung der Reichsschrifttumskammer, die unter anderem die „Liste des unerwünschten und schändlichen Schrifttums“ herausgab. Die Reichsschrifttumskammer war ein Instrument zur Kontrolle von Schriftstellern und Verlegern. Ihren Mitarbeitern oblag es, Schriftsteller in die Kammer aufzunehmen oder ihnen mangels „Eignung“ (etwa bei jüdischer Herkunft, Mitgliedschaft in einer kommunistischen Vereinigung oder Homosexualität) die Mitgliedschaft zu verweigern (vgl. Barbian 2010). Dies kam einem Berufsverbot gleich. Zudem überwachte die Kammer deutsche Verlage: Die Rechtsgrundlage hierfür war die am 4. Februar 1933 von Hindenburg erlassene „Verordnung zum Schutze des deutschen Volkes“, durch die sämtliche „Druckschriften, deren Inhalt geeignet ist, die öffentliche Sicherheit oder Ordnung zu gefährden“ (Reichsgesetzblatt vom 1933; vgl. auch Barbian 2010: 27) eingezogen werden konnten. Diese Verordnung blieb bis 1945 die Rechtsgrundlage für die Beschlagnahmung von Büchern, die nicht der Propaganda nationalsozialistischer Grundwerte dienten.

Das Leben des Menschen wurde 1933 von der Gestapo aus dem Verlagshaus Franckh beschlagnahmt. Aus historischer Perspektive hatten Verlage mehrere Möglichkeiten, von den Machthabern nicht zur Veröffentlichung freigegebene Werke weiter zu publizieren: Bücher wurden einer internen Zensur unterzogen und kritische Passagen gestrichen oder Verleger einigten sich durch direkten Kontakt mit der Reichsschrifttumskammer über die Möglichkeit einer weiteren Verwendung der fraglichen Bücher. Im Fall von Das Leben des Menschen entsteht durch die Quellen der Eindruck, dass Franckh sich zunächst recht unbeeindruckt von der NS-Gesetzgebung gezeigt hat: Im Kosmos-Taschenkalender für die deutsche Jugend des Jahres 1934/1935 findet sich mit „Das Gruseln von Dr. Fritz Kahn“ ein illustrierter Artikel, den Kahn bereits 1922 in Wunder in uns veröffentlicht hatte. ${ }^{30}$ Die in Deutschland unverkäuflichen Exemplare von Kahns Buchreihe wurden, wie eine Werbung verrät, ${ }^{31}$ bis 1935 in das deutschsprachige Ausland verkauft - ein Befund, der mit dem historischen Höhepunkt der Buchexportförderung als Mittel der Auslandspropaganda zusammenfällt (vgl. Barbian 2010: 121-122). 
Aufgrund seiner jüdischen Herkunft konnte Fritz Kahn die Originalwerke nicht mehr in Deutschland veröffentlichen. Die Quellen belegen jedoch, dass Franckhs Interesse an einer Weiterverwendung so groß war, dass der Verlag in direkten Kontakt mit der Reichsschrifttumskammer trat - ein durchaus übliches Vorgehen (vgl. Barbian 2010: 420). Zuvor nahm der Verlag erneut Kontakt zu den Illustratoren auf, um zu klären, welche Rolle sie bei der Entstehung der Illustrationen in Das Leben des Menschen gespielt hatten. Die schriftliche Anfrage an Roman Rechn ist nicht mehr erhalten, wohl aber dessen Antwort vom 5. Juni 1936:

\begin{abstract}
Zur Frage der Urheberschaft „meiner“ Bilder im „Leben des Menschen“ kann ich nur Folgendes sagen: dass diese, vom ersten bis zum letzten in der Idee und auch im Aufbau von mir entworfen worden sind. [...] So hat auch Herr Dipl. Ing. Fritz Schüler „seine“ Tafeln und Bilder ideenmäßig vollkommen selbstständig freischöpferisch geschaffen; vom „Mensch als Industriepalast“ bis zum „Blutkreislauf". Dr. Kahn gab uns lediglich das Thema und stand uns als scharfer Kritiker zur Seite oder gegen uns. Er war ja gar nicht in der Lage, auch nur die primitivste Skizze zu Papier zu bringen; als spitzer Analytiker in seiner Kritik zwang er uns gerade dadurch zu nie rastender Tätigkeit des Geistes. Nicht die Ausführung einer Zeichnung dauerte daher oft wochenlang. sondern die Durcharbeit immer neuer Ideen zu einem Problem. [...] Dr. Kahn gab uns das Thema - die Ideen aber hatten wir. ${ }^{32}$
\end{abstract}

Dieses Dokument ist, ähnlich wie die Antworten Fritz Schülers und Otmar Tresters auf Franckhs Anfrage wegen der Verpflichtungsscheine aus dem Jahr 1931, ein Hinweis darauf, dass die Zeichner sich als eigenständig schaffende Akteure sahen. Im historischen Kontext des Jahres 1936 wird an diesem Brief aber ebenso deutlich, dass durch Rechns Brief Fritz Kahns Rolle bei der Entstehung von Das Leben des Menschen marginalisiert werden sollte. Möglicherweise führte Franckh dies Argument gegenüber der Reichsschrifttumskammer an, als es um die Weiterverwendung von Kahns Werk ging.

Im Oktober nahm Franckh über Hans Eberhard Günther Kontakt zur Reichsschrifttumskammer auf. Aus einem Dokument geht hervor, dass Günther bei einem „Herrn Menz" vorgesprochen hatte. Es ist anzunehmen, dass es sich bei „Herrn Menz" um den Kammerreferenten Herbert Menz handelte, der seit Anfang 1936 als rechte Hand des Reichskulturkammerpräsidenten Hanns Johst tätig war (Barbian 2010: 149) und über weitreichende Kompetenzen sowie eine ausgezeichnete Kenntnis der deutschen Verlagslandschaft verfügte (ebd.: 420, 424). Menz formulierte die Bedenken der Reichsschrifttumskammer gegen die Weiterverwendung von Kahns Bildern, Günther konnte aber erreichen, dass

Franckh die 40 Exemplare [behält, M.E.]. Sie werden nummeriert, sodass der Bestand kontrolliert werden kann. Es ist Franckh gestattet, die neuen Autoren mit dem Kahnschen Werk zu versehen. [...] Es ist aber in jedem dieser Fälle die Erlaubnis der RSK [Reichsschrifttumskammer, M.E.] einzuholen. [...] Es ist nun 
wohl nicht mehr nötig, dass Sie aus einem Teil dieser 40 Ex. die Tafelbilder ausschneiden. $^{33}$

Die hier aufscheinende Strategie, ein Werk anhand der Verbindung zwischen Schriftsteller und dessen vermeintlich ,arischem “ oder ,jüdischem “ Charakter zu bewerten, führte so weit, dass in verlagseigenen Karteien die Bilder aus Das Leben des Menschen einer rassentheoretischen Differenzierung unterzogen wurden. In einer Bibliographie ist über Das Leben des Menschen vermerkt:

Wurde 1933 von der Gestapo beschlagnahmt. Schaubilder „Der Mensch als Industriepalast“ u. „Stammbaum des Menschen“ auch apart erschienen in der Reihe „Kosmos-Wandbilder“. [...] Die Rechte der Schautafeln - von jüdischem Geist ersonnen, aber von arischen Künstlern ausgeführt - blieben auch nach der Beschlagnahmung bei Franckh. ${ }^{34}$

Auch Kahn blieb die Trennung von Autor und Werk sowie der Plan zur Neuveröffentlichung nicht verborgen. Er selbst wollte die Bücher im Exil neu veröffentlichen und seinen Namen mit dem Werk enger verbinden. Im September 1936 war Kahn aus Palästina nach Europa zurückgekehrt und hielt sich in Liechtenstein auf. Hier plante er die Neuauflage der Bücher in der Schweiz und die Übersetzung seiner Werke für den US-amerikanischen Markt. Für diese Veröffentlichungen brauchte er die Platten und Klischees sowie das Recht zur Veröffentlichung - beides befand sich im Besitz von Franckh. Auf die Rückgabe der Verlags- und Urheberrechte konnten beide Parteien sich im September 1936 einigen. Die Platten und Klischees, sowie die Bildrechte gab der Verlag zunächst nicht zurück. ${ }^{35}$ Doch im Februar 1937 willigte die Franckh'sche Verlagshandlung ein, „daß sie diese Rechte an den Bildern für die im Ausland erscheinenden Ausgaben vom Leben des Menschen Herrn Dr. Fritz Kahn überlässt". 36

Damit waren ab 1937 die Illustrationen aus Fritz Kahns Büchern vollständig aus ihrem Ursprungskontext herausgelöst und konnten in neue, politisch unterschiedlichste Kontexte überführt werden: in die Schweiz, die USA und das nationalsozialistische Deutschland. Die Produktion der neuen Bücher erfolgte in anderen Kollektiven und die Veröffentlichung von Bildmaterial aus einem Buch, das verboten worden war, ${ }^{37}$ tradierte eine seit fast zwei Jahrzehnten bestehende visuelle Kultur, indem das Bildmaterial Eingang fand in andere visuelle Kulturen und diese wiederum prägte.

\section{Alte Bilder in neuen Büchern I: Das Leben des Menschen im Nationalsozialismus}

Nachdem das Buch unter Kahns Autorschaft im Nationalsozialismus nicht mehr publiziert werden konnte und Text und Bilder voneinander getrennt worden waren, bestand die Möglichkeit, einen Teil der Illustrationen 
anderweitig zu publizieren. Dafür benötigte man einen neuen Autor und Gerhard Venzmer erwies sich als geeignet. Er hatte in Hamburg Medizin und Philosophie studiert und in beiden Fächern promoviert. Seine Tätigkeit als Autor für die Franckh'sche Verlagshandlung nahm er 1926 auf (Harten et al. 2006: 482). Bis 1933 waren seine Veröffentlichungen mit Bildern aus Das Leben des Menschen illustriert und dies durch die Bildunterschrift „Aus Kahn, Leben des Menschen“ kenntlich gemacht (Venzmer 1930a, 41-47). Ab 1936 wurden die Bilder mit den Namen der Verlagszeichner signiert und nach 1938 mit der Bildunterschrift „Bildarchiv Franckh“ versehen. Die Themen von Venzmers Kosmos-Bändchen verwiesen bereits vor 1933 im Rahmen eines normativen Krankheits- und Gesundheitsverständnisses auf die Verschränkung von äußerlicher Gestalt oder Stoffwechseltypus und charakterlichen Eigenschaften (vgl. Stoff 2008), wie sich an Titeln wie Körpergestalt und Seelenanlage (Venzmer 1930b) oder Deine Hormone - Dein Schicksal! (Venzmer 1933) ablesen lässt. ${ }^{38}$

Nach der Machtübernahme der Nationalsozialisten trat Venzmer sowohl in die NSDAP als auch die SA ein (Harten et al. 2006: 482). Bei Franckh war er als Ressortleiter seit 1942 für den medizinischen Inhalt des Kosmos verantwortlich ${ }^{39}$ und veröffentlichte die beiden Bücher Der Mensch und sein Leben Ein Volksbuch vom menschlichen Körper (1938) sowie das Hand-und Lehrbuch der Krankenpflege (1938/1939). ${ }^{40}$ Erstgenanntes Werk war eine einbändige Zusammenfassung von Das Leben des Menschen. Doch nicht nur die Nominalphrase des Buchtitels war umgestellt worden, sondern auch inhaltlich war Kahns Werk mit der nationalsozialistischen Gesundheitspropaganda gleichgeschaltet worden - und dies nicht nur in dem neu hinzugefügten Kapitel „Rassenkunde und Rassenpflege“ .

Die Bilder aus Das Leben des Menschen kamen vor allem im ersten Teil des Werkes vor. Venzmer relativierte im Vorwort von Der Mensch und sein Leben nicht nur den Vergleich zwischen Mensch und Technik, sondern grenzte es von dessen Vorgänger auch dadurch $a b$, dass er die Bilder als alleiniges Eigentum der Franckh'schen Verlagshandlung deklarierte und zugleich ein neues Verständnis beim Gebrauch von Technikanalogien verdeutlichte:

Dabei wurde ganz besonderer Wert auf eine Bebilderung gelegt, die imstande sein sollte, das Gesagte im besten Sinne des Wortes anschaulich zu machen; und hierbei kamen dem Buch ganz besonders die reichhaltigen Bildbestände aus dem Archiv der Franckh'schen Verlagshandlung zugute, [...]. Bei der Anfertigung aller dieser Illustrationen wurde bisweilen auch nicht davor zurückgeschreckt, Tätigkeiten des Körpers mit diesen oder jenen Vorgängen aus dem Reiche der Technik in Parallele zu setzen; und dies geschah aus der sicheren Überzeugung, daß kein einsichtiger Leser aus solchen Gegenüberstellungen falsche Schlüsse ziehen kann (Venzmer 1940, Vorwort).

Für Venzmers Ausgabe wurden vor allem die anatomischen Zeichnungen übernommen. Unter den Bildern befand sich zwar Arthur Schmitsons 
Darstellung des Herzens, es fehlten aber Bilder, die eine kunstvolle Darstellung des Menschen zeigten, wie sie etwa in den Reiseerlebnissen einer Wanderzelle zum Tragen gekommen war. Und obwohl Venzmer sich alle Mühe gab, Das Leben des Menschen mit seinem Buch zu überschreiben, fanden sich in den Signaturen der Bilder überraschende Verweise auf das Buch: Franckh übernahm die Druckplatte mit der anatomischen Darstellung des Herzens aus Kahns Buch. Der Verlag hatte sich mit Kahn darauf verständigt, die Bilder aus seinem Werk mit „neutralen Bildunterschriften“ zu versehen und wies das Bild entsprechend als aus dem „Verlagsarchiv Franckh“ stammend aus. ${ }^{41}$ Doch obwohl neutrale Bildunterschriften gewählt wurden, waren die Signaturen der Illustratoren, wie etwa Arthur Schmitsons, nicht unkenntlich gemacht worden, die so auf Das Leben des Menschen zurückweisen.

Venzmers Der Mensch und sein Leben lässt sich als das Ergebnis eines transkriptiven Vorganges interpretieren. Durch die Gesetzgebung im Nationalsozialismus war Das Leben des Menschen verboten, Kahns Buch sollte unlesbar gemacht werden. Für eine Neuveröffentlichung im NS griff Franckh auf einzelne Bestandteile aus Kahns Werk zurück, im Sinne Jägers könnte man dies als Erhaltung der Lesbarkeit der älteren semantischen Textur deuten (Jäger 2004a: 73): Fritz Kahns Veröffentlichungen wurden mittels Fritz Kahns Veröffentlichungen überschrieben.

\section{Alte Bilder in neuen Büchern II: Das Leben des Menschen im Exil}

„Transkribierende Texturen können jederzeit ihrerseits wieder transkribiert werden, wie umgekehrt transkribierte Texturen erneut als Transkriptionen fungieren können. Lesbarkeit und Unlesbarkeit sind Eigenschaften, die nur relativ zu bestimmten kommunikativen Situationen zugeschrieben werden dürfen“ (Jäger 2004a: 73). In Anlehnung an Jäger untersucht der folgende Abschnitt, wie Kahn Das Leben des Menschen im Exil umschrieb und in neue Richtungen entfaltete, denn auch Kahn rekontextualisierte die Bilder. Dabei liegt wiederum ein besonderes Augenmerk auf dem Gebrauch und der Funktion von Signaturen. Bis 1943 waren seine wichtigsten Exilveröffentlichungen erschienen: Der Mensch gesund und krank (Kahn 1939, Schweiz) sowie Der Mensch, Bau und Funktionen unseres Körpers (Kahn 1940, Schweiz) und schließlich das amerikanische Man in Structure and Function (Kahn 1943b, USA). Alle Bücher sind weniger umfassend als Das Leben des Menschen. An der Signatur der Illustrationen lässt sich zeigen, wie Autorname und Werk enger verknüpft werden.

Kahn hatte nur wenige Originalbilder ins Exil retten können. Als inzwischen erfahrener Autor kannte er aber die technischen Möglichkeiten, Bilder 
in Drucktafeln zurück zu überführen. ${ }^{42}$ Diese Bilder zeigen, wie seine Publikationen aus der Weimarer Republik neuen Kontexten angepasst werden konnten. Im Unterschied zu den Veröffentlichungen, die Übersetzungen von Das Leben des Menschen darstellten, und anders als Venzmer, der nur auf das vorhandene Bildmaterial aus Das Leben des Menschen zurückgriff, entwickelte Kahn den visuellen Stil in der Zusammenarbeit mit einem neuen Kollektiv an Zeichnern im Exil weiter. Im Hinblick auf die Autorisierung der Bilder, die er als sein eigenes Werk erachtete, verfuhr Kahn im Umgang mit den Bildern anders als das Verlagshaus Franckh: Es findet sich keine Spur mehr, die auf die Genealogie der Bilder verweist. Das Menschenherz und die Reiseerlebnisse einer Wanderzelle von Arthur Schmitson wurden für Der Mensch und Man in structure and function originalgetreu reproduziert, Schmitsons Signatur aber retuschiert.

Besonders hervorzuheben ist, dass die Bilder in den USA nicht nur als populäre medizinische Bilder wahrgenommen wurden. Als das Sunday Mirror Magazine (Anonym 1943) Kahns Man in structure and function dem amerikanischen Publikum präsentierte, zierte Arthur Schmitsons Herz, das auf eine mikroskopische Abbildung von Herzmuskelzellen montiert war, die erste Seite des Artikels. Der Rezensent bezeichnete es als verwandt mit dem Genre des Surrealismus oder der abstrakten Kunst und verglich es mit dem Werk des Architekten Frank R. Pauls (1884-1963), der in den USA ein Pionier der Science-Fiction Zeichnung war. Die Bilder von Arthur Schmitson, einem Maler, dessen Karriere mit den Zeichnungen für einen Anatomieatlas in einem fachwissenschaftlichen Kontext begonnen hatte und der diese in den 1910er Jahren mit den Illustrationen für populäre medizinische Bücher fortsetzen konnte, wurden 25 Jahre später in modifizierter Form einem internationalen Publikum als surrealistische Darstellung präsentiert und dem Genre des Science Fiction zugewiesen. Dem Bild fehlte jedoch eine Signatur, die auf seinen Ursprungszusammenhang hätte hinweisen können. Statt der Signaturen der Zeichner fand sich fortan ein Schnipsel mit Fritz Kahns Initialen und einem Copyrightzeichen, das nun in eine Ecke des Bildes geklebt wurde und Kahn als einzigen Produzent und Eigentümer auswies. In der Exilzeit wurden Bild und Text noch stärker als in den 1920er Jahren ausschließlich mit dem Autor verknüpft.

Ging es in den 1920er Jahren darum, eine festgelegte Erscheinungsweise visueller Aufklärungskultur zu charakterisieren und zu etablieren, so ging es nun darum, diese Visualität zu einem Stil zusammenzufassen und sie gegen andere Visualisierungsformen (wie bei Venzmer) über die Marke „Fritz Kahn“ (vgl. Borck 2007: 507) abzugrenzen. Dies steht im Gegensatz zu Das Leben des Menschen, in dem eine Vielzahl von Signaturen zu verzeichnen gewesen war. 


\section{Signaturen als Spuren veränderten Bildgebrauchs}

Populäre Schriften wie Das Leben des Menschen trugen in der Weimarer Republik dazu bei, dass eine breite Bevölkerungsschicht Kenntnis über Bau und Funktion des menschlichen Körpers erlangte. Kahns Buchreihe war hierzu ein Mittel, das sich aufgrund seiner innovativen Illustrationen außerordentlicher Beliebtheit erfreute und einen hohen Bekanntheitsgrad erlangte.

Die nähere Untersuchung der Entstehungsgeschichte hat gezeigt, wie Das Leben des Menschen von anderen Quellen wie der zeitgenössischen Publikation Wunder in uns ebenso wie der anatomischen Atlaszeichnung, der Ingenieurszeichnung und der Werbegraphik beeinflusst wurde. War Das Leben des Menschen bereits das Ergebnis einer Transkription populärer Motive des Körpers, wurde es nun selbst Gegenstand eines transkriptiven Verfahrens. Nach dem Ende der Weimarer Republik und dem Verbot der Buchreihe im nationalsozialistischen Deutschland fanden ihre Inhalte und vor allem die Illustrationen Eingang in unterschiedlichste Kontexte. Wie diese Einbettung geschah, liess sich anhand der nachgelassenen Korrespondenz zwischen Franckh und der Gestapo einerseits sowie Franckh und Kahn andererseits nachvollziehen.

Noch eindrücklicher als die Briefwechsel allerdings zeigen Bildsignaturen den Wechsel zwischen Beweglichkeit und Fixierung bildlicher Objekte an. Sie verdeutlichen, wie ein Motiv aus einem Prätext (im Sinne Jägers) transkribiert und schließlich zu einem Skript wird. Dabei führt das neue Skript Spuren auf den Prätext mit sich, die vor allem in der historischen Analyse sichtbar werden: Die Illustrationen stellen den menschlichen Körper dar, während die Signaturen als Zeichen einer neuen Rahmung über das Bildmotiv hinausweisen (vgl. Jäger 2004a: 76). Die Analyse von Illustration und Signatur gibt nicht nur Auskunft über die Spezifik der populären Darstellung in der medizinischen Aufklärungsliteratur und ihren Veränderungsformen von Kahn zu Venzmer, sondern darüber hinaus auch über die komplexen Produktionsbedingungen und die Frage nach Autorschaft und Autorisierung, das heißt über die Bestrebungen von Vereinheitlichung bzw. Trennung von Autor und Werk.

Die Franckh'sche Verlagshandlung versuchte, die Bilder in ihrem neuen Kontext zu fixieren, indem sie sie aus dem Verlagsarchiv Franckh stammend auswies. Da aber die Originalsignaturen aus Das Leben des Menschen ebenfalls sichtbar blieben, blieb auch der Prozess der Überschreibung und der Prätext erkennbar. Im Exil klebte Kahn ein Copyrightzeichen auf die Druckplatten, nachdem die Signaturen der Zeichner aus den 1920er Jahren retuschiert worden waren. Anders als Franckh verwies er bei der Einführung von Man in structure and funtion auf den US-amerikanischen Markt auf sein Werk aus den 1920er Jahren (Kahn 1943a). Für die historische Analyse erweist sich der Aufkleber mit Namen und Copyrightzeichen nicht nur als zusätzliche 
materielle Schicht, sondern erlaubt eine genauere Bestimmung des Verhältnisses von Bild und Signatur.

In beiden Fällen ist zu konstatieren, dass die Bilder überaus mobil und unabhängig von ihrem Ursprungskontext einsetzbar waren. Bei Bildern, die in sich diametral gegenüberstehenden Kontexten auftauchen, könnte es bisweilen den Anschein haben, dass die Bilder geradezu ein Eigenleben führen: Ein Bild, das ausgeschnitten und in neue Bücher eingefügt wird, wandert zwischen Zeichnern und Schreibern, Verlagen und Institutionen hin und her. In der Rückbindung an diejenigen, die die Illustrationen ausgedacht, hergestellt und rekontextualisiert haben, lässt sich das Eigenleben der Bilder, wie der Bildwissenschaftler Walter Mitchell es ausdrückt, besser verstehen (Mitchell 2008). Die Signaturen verweisen letztlich auch darauf, dass Bilder Personen brauchen, die sie bearbeiten und in neue Kontexte einbinden. Sich auf die Spuren von Illustrationen, Signaturen und Personen zu begeben, trug zum Verständnis der Produktion und Transkription von Fritz Kahns Werk an den Übergängen von Weimarer Republik, Nationalsozialismus und Exil bei.

\section{Danksagung}

Das Archivmaterial für diesen Beitrag stammt aus dem Nachlass von Fritz Kahn im Leo Baeck Institut (New York) sowie dem Archiv des FranckhKosmos Verlags (Stuttgart). Ich danke insbesondere Elke Rutschmann und Zachary Loeb für die Unterstützung meiner Archivarbeit sowie Günther Rechn (Cottbus) und Martina Rechn (Ludwigshafen) für ihre Bereitschaft, Auskunft über das Leben ihres Onkels bzw. Großonkels Roman Rechn zu geben. Für hilfreiche Kommentare und konstruktive Kritik zu einer früheren Version dieses Artikels danke ich drei anonymen Gutachtern/Gutachterinnen. Für die Genehmigung zum Abdruck der Abbildungen eins bis vier danke ich Thilo von Debschitz und der Abbildungen fünf bis acht dem Leo Baeck Institut.

\section{Anmerkungen}

1 Aus der hier dargestellten Skizze wird bereits deutlich, dass es in diesem Beitrag nicht um die Frage nach Interpretation der Bilder mit Blick auf das Menschenbild von der Weimarer Republik hin zum NS gehen wird. Damit steht auch die Rekontextualisierung von Bildern durch ihre sprachliche Rekontextualisierung nicht im Vordergrund - auch wenn diese sicher ebenfalls Gegenstand einer Untersuchung transkriptiver Verfahren sein könnten.

2 Kosmos-Bändchen waren Taschenbücher mit einem Umfang von ca. 100 Seiten, die ein naturkundliches Thema stärker als der Kosmos-Handweiser vertieften. Sie wurden mehrmals pro Jahr an die Mitglieder der „Gesellschaft der Naturfreunde“ verschickt. 
3 Zitat aus der Verlagsbroschüre: Kosmos-Geschichten. 175 Jahre Kosmos-Verlag, S. 6, Archiv der Franckh-Kosmos GmbH \& Co. KG Stuttgart (kurz: Archiv Franckh-Kosmos).

4 Walter Widmann, Hinter den Kulissen eines naturwissenschaftlichen Verlags, FranckhErinnerungen 1916-1963, unveröffentlichtes Manuskript, Archiv Franckh-Kosmos.

5 Ebd.

6 Werkvertrag zwischen Fritz Kahn und der Franckh'schen Verlagshandlung, unterzeichnet im August 1912, Umschlag VII: Alte Verträge, Archiv Franckh-Kosmos.

7 Diese Information geht aus einem Zusatzvertrag hervor, der im Juni 1914 unterzeichnet wurde und den Werkvertrag von 1912 ergänzte. Paragraph drei regelte Fritz Kahns Zuständigkeit für das Abbildungsmaterial in der geplanten Veröffentlichung, Umschlag VII: Alte Verträge, Archiv Franckh-Kosmos.

8 Franckh'sche Verlagshandlung an Fritz Kahn, 3.8.1914, Archiv Franckh-Kosmos.

9 Ebd.

10 Dieser Werkvertrag vom 16.2.1921 ersetzte den Werkvertrag von 1912 und den Zusatzvertrag von 1914, Umschlag IX, Archiv Franckh-Kosmos.

11 Ebd.

12 Ebd.

13 Dies belegt ein Zeitungsausschnitt über die statistische Benutzung der Bibliotheksbücher. Das Dokument ist undatiert. Im Nachlass ist der Ausschnitt in ein Sammelalbum eingeklebt. Allem Anschein nach klebte Fritz Kahn in dieses Buch Dokumente, die die Bedeutung seiner Bücher besonders gut dokumentierten. Neben dieser Notiz finden sich noch weitere Zeitungsausschnitte und Rezensionen, Leserbriefe, ein Gratulationsschreiben der Franckh'schen Verlagshandlung zur Fertigstellung der Buchreihe sowie Werbung, alle Dokumente in Arthur and Fritz Kahn Collection, AR 7144/MF 746, Box 1, Folder 57. Leo Baeck Institute New York (kurz LBI).

14 Vgl. hierzu: Leserbrief des Redakteurs der Pastoralblätter (1923); Brief aus der Pressestelle des Preußischen Staatsministeriums (1926); Programm eines Fritz-Kahn-Abends der Wissenschaftlichen Studiengemeinschaft Mittweida (1927); Zuschrift eines PsychoPhysiognomikers (1928), alle Dokumente in Arthur and Fritz Kahn Collection, AR 7144/MF 746, Box 1, Folder 57, LBI.

15 Rezension in der Vossischen Zeitung am 14.8.1932.

16 Fritz Kahn an Richard Holzwarth, 28.6.1938, Umschlag X: Kahn - Differenzen, Archiv Franckh-Kosmos.

17 Ich danke Birgit Nemec für einen Vorabdruck ihres Artikels Anatomical Modernity in Red Vienna: Julius Tandler's Textbook for Systematic Anatomy and the Politics of Visual Milieus, der 2015 in Sudhoffs Archiv erscheinen wird.

18 Anja Casser hat auf die enge Verbindung zwischen technischer Berufsausbildung und spezifischer Bildsprache populärer Bilder hingewiesen. Sie zeigt am Beispiel der Graphiken, die im Atelier der Brüder von Römer in München entstanden sind, wie die Berufsausbildung der Brüder als Architekt bzw. Ingenieur deren ingenieurtechnische Graphiken prägte, die sie eigens für populäre Publikationen über Luft- und Raumfahrt herstellten (vgl. Casser 2007: 113-136).

19 Die Dokumentation der Schülerschaft des Staatlichen Bauhaus Weimar verzeichnet, dass Roman Rechns Bewerbung für ein Studium bei Walter Gropius abgelehnt wurde: Probeweise Aufnahme und abgelehnte Gesuche um Aufnahme sowie zurückgezogene Anmeldungen von Schülerinnen bzw. Schülern, Aktennummer 161, Laufzeit: $(1908,1916)$ 1919-1925, Staatliches Bauhaus Weimar, Thüringisches Hauptstaatsarchiv Weimar. Für den Hinweis auf dieses Dokument danke ich Michael Sappol.

20 Manuskripte 1930s-1967 Arthur and Fritz Kahn Collection, AR 7144/MF 746, Box 2, Folder 41-Box 3, Folder 40, LBI.

21 Undated. Arthur and Fritz Kahn Collection, AR 7144/MF 746. Box 3, Folder 23, LBI.

22 Dass Kahn seine Illustratoren persönlich anleitete und überdurchschnittliche zeichnerische Qualität einforderte, geht aus einem Schriftwechsel zwischen der Franckh'schen Verlagshandlung und Fritz Kahn hervor. Nachdem Kahn die Zusammenarbeit mit einem Zeichner abgelehnt hatte, bemerkte der Verlag: „Auf Elser möchten wir doch noch einmal 
hinweisen. Was andere sich erst ganz mühsam sich (sic) angeeignet haben und teilweise erst durch Ihre Anleitung erreichen, das hat doch Elser von sich aus recht gut erarbeitet und wenn er auch jetzt, wie Sie schreiben, nicht über das Mittelmass hinausreicht, so ist er (sic) doch wahrscheinlich, da er das alles allein geschafft hat, leicht weiterzuentwickeln und über andern (sic) Durchschnitt hinaus fortzubilden“. Franckh'sche Verlagshandlung an Fritz Kahn, 26.9.1922, Umschlag IX, Archiv Franckh-Kosmos.

23 Arthur and Fritz Kahn Collection, AR 7144/MF 746, Box 2, Folder 6 and OS43, LBI.

24 Arthur and Fritz Kahn Collection, AR 7144/MF 746, Box 2, Folder 7 f., LBI.

25 Der den Verpflichtungsschein begleitende Brief ist nicht erhalten. Das Antwortschreiben von Fritz Schüler an die Franckh'sche Verlagshandlung gibt einen Hinweis auf den Inhalt dieses Schreibens: „Den Verpflichtungsschein kann ich in dieser Form nicht unterschreiben, - Ich kann mich schriftlich verpflichten, Zeichnungen die ich für Ihren Verlag bei Dr Kahn anfertigte nicht anzubieten oder zu veräussern" [im letzten Satz fehlt die Verneinung]. Schüler an die Franckh'sche Verlagshandlung, 19.6.1031, Umschlag V: Zeichner - Mitarbeiter, Archiv Franckh-Kosmos.

26 Brief mit unterzeichnetem Verpflichtungsschein von Roman Rechn an die Franckh'sche Verlagshandlung, 22.5.1931, Umschlag V: Zeichner - Mitarbeiter. Archiv FranckhKosmos.

27 Fritz Schüler an die Franckh'sche Verlagshandlung, 19.6.1931, Umschlag V: Zeichner Mitarbeiter, Archiv Franckh-Kosmos.

28 Franckh'sche Verlagshandlung an Fritz Kahn, 16.12.1931, LBI, Arthur and Fritz Kahn Collection, AR 7144/MF 746, Box 1, Folder 57, LBI.

29 Ebd.

30 Dieser Artikel war bereits eine Wiederholung, denn „Das Gruseln“ war bereits 1920 im Kosmos erschienen, siehe Kahn 1920: $12 \mathrm{f}$.

31 Archivalie, Werbekarte. Kosmos XXXII, 1935. Archiv Franckh-Kosmos.

32 Roman Rechn an Franckh'sche Verlagshandlung, 5.6.1936, Umschlag V, Archiv FranckhKosmos.

33 Hans Eberhard Günther an Franckh'sche Verlagshandlung, 1.10.1936, Umschlag X, Archiv Franckh-Kosmos.

34 Bibliographie des Verlagshauses, die auf Karteikarten geführt wurde, ca. 1940, Archiv Franckh-Kosmos.

35 Franckh'sche Verlagshandlung an Fritz Kahn, 20.09.1936, Umschlag IX, Archiv FranckhKosmos

36 Erklärung der Franckh'schen Verlagshandlung, 19.02.1937, Umschlag IX, Archiv FranckhKosmos.

37 „Der Verkauf wurde verboten, alle erreichbaren Exemplare wurden eingezogen und in Papiermühlen vernichtet", erinnerte sich Kahn nach dem Ende des Zweiten Weltkriegs. Ich danke Tilo Krause für die deutsche Übersetzung des Interviews En forfatter soger seks personer ..., das die dänische Zeitung Berlingske Tidende 1960 mit Kahn führte.

38 Zur Darstellung der Physiologie als industriellen Prozess siehe Stoff 2008.

39 Kurt Fleischmann an die Schriftleiter des Kosmos, 27.11.1942, Ordner Kosmos I, Archiv Franckh-Kosmos.

40 Bei dem Handbuch der Lehr-und Krankenpflege handelte es sich um eine Neuherausgabe eines Teils von Der Mensch und sein Leben. Beide Veröffentlichungen stimmen überein, weshalb ich im weiteren Text lediglich Der Mensch und sein Leben als Untersuchungsgegenstand herangezogen habe.

41 Die Franckh'sche Verlagshandlung behielt sich das Recht vor, die Bilder aus Das Leben des Menschen zu veröffentlichen, allerdings ohne Herkunftsnachweis. In einem Schreiben an Fritz Kahn vom 1.6.1938 sicherte sie zu, dass „Voraussetzung für die Verwendung ist, dass sie Abbildungen nicht als Erzeugnis eines anderen Urhebers bezeichnet, sondern mindestens neutrale Unterschriften gewählt werden“. Auf dem Briefbogen ist zusätzlich handschriftlich vermerkt: „also auch Kosmos Kalender etc. immer mit d. Unterschrift Bildarchiv Franckh“, siehe Umschlag X, Archiv Franckh-Kosmos.

42 Fritz Kahn an Walther Keller, 2.4.1937, Umschlag X, Archiv Franckh-Kosmos. Kahn schreibt: „Bestehen Möglichkeiten, dass Sie Galvanos nach USA? Ich kann sie natürlich 
herstellen lassen, es ist weder schwer, die Originale zu kopieren und neue Chlichés zu machen, noch nach gedruckten Vorlagen mit Retusche direkt Galvanos herstellen zu lassen."

\section{Literatur}

Ahren, Eva und Michael Sappol 2009. The Strange Space of the Body: Two Dialouges. In: André Jansson und Amanda Lagerkvist (Hg.). Strange Spaces. Explorations into Mediated Obscurity. Aldershot: Ashgate: 73-100.

Anonym 1943. Pen-Rays. Sunday Mirror Magazine: Section 6-7.

Azzouni, Safia 2015. Autorität und Autorschaft in Paratexten der Populärwissenschaft. In: Safia Azzouni, Stefan Böschen und Carsten Reinhardt. Erzählung und Geltung. Wissenschaft zwischen Autorschaft und Autorität. Weilerwist: Velbrück Wissenschaft: 155-172.

Barbian, Jan-Pieter 2010. Literaturpolitik im NS-Staat. Von der „Gleichschaltung“ bis zum Ruin. Frankfurt am Main: Fischer.

Borck, Cornelius 2007. Communicating the Modern Body: Fritz Kahn Popular Images of Human Physiology as an Industrialized World. Canadian Journal of Communication (35): 495-520.

Borck, Cornelius 2009. Bild der Wissenschaft. Neuere Sammelbände zum Thema Visualisierung und Öffentlichkeit. NTM. Zeitschrift für die Geschichte der Wissenschaften, Technik und Medizin (17): 317-327.

Bölsche, Wilhelm 1913. Wie und warum soll man die Naturwissenschaft ins Volk tragen? Nachdruck aus ders.: Stirb und Werde. Naturwissenschaftliche Plaudereien. Jena: Diederichs. Arbeitsblätter für die Sachbuchforschung 14, 2007. URL: http://www.sachbuchforschung. uni-mainz.de/wp-content/uploads/Arbeitsblaetter_Sachbuchforschung_14.pdf (Stand: 09.03.2014).

Bölsche, Wilhelm 1898-1903. Das Liebesleben in der Natur. 3 Bde. Leipzig: Diederichs.

Bourgery, Jean Baptiste Marc et Nicolas Henri Jacob 1832-1854. Traité complete de l'anatomie de l'homme comprenant la médicine operatoire. Avec planches lithografiées d'après nature par H. Jacob. 8 Bde. Paris.

Daston, Lorraine und Peter Galison 2007. Objektivität. Frankfurt am Main: Suhrkamp.

Daum, Andreas 2002. Wissenschaftspopularisierung im 19. Jahrhundert: Bürgerliche Kultur, naturwissenschaftliche Bildung und die deutsche Öffentlichkeit, 1848-1914. München: Oldenbourg.

Debschitz von, Uta und Thilo von Debschitz 2009. Fritz Kahn: man machine/Maschine Mensch. Wien: Springer.

Döblin, Alfred 1922. Metapsychologie und Biologie. Neue Rundschau, 12, 1231-1232.

Draaisma, Douwe 1999. Die Metaphernmaschine. Darmstadt: Wissenschaftliche Buchgesellschaft.

Eilers, Miriam 2012. Urbane Natur und Kultur - Die Stadt Berlin der 1920er Jahre reflektiert im Werk Fritz Kahns. In: Johanna Bleker, Marion Hulverscheidt und Petra Lennig (Hg). Visiten. Berliner Impulse zur Entwicklung der modernen Medizin. Berlin: Kadmos: 197-212.

Francé, Raoul H. 1921. Das Leben der Pflanze. Stuttgart: Franck'sche Verlagshandlung.

Gall, Alexander 2011. Wunder der Technik, Wunder der Natur. Zur Vermittlung eines medialen Topos. In: Alexander C. T. Geppert und Till Kössler (Hg.). Wunder. Poetik und Politik des Staunens im 20. Jahrhundert. Berlin: Suhrkamp: 270-301.

Günther, Hans 1921. Wunder in uns. Zürich: Rascher.

Harten, Hans-Christian, Uwe Neirich und Matthias Schwerendt 2006. Rassenhygiene als Erziehungsideologie des Dritten Reichs. Berlin: Akademie Verlag.

Heumann, Ina und Axel C. Hüntelmann 2013. Einleitung: Bildtatsachen. Visuelle Praktiken der Wissenschaften. Berichte zur Wissenschaftsgeschichte (36): 283-293.

Heumann, Ina 2014. Gegenstücke. Populäres Wissen im transatlantischen Vergleich (1948-1984). Wien: Böhlau.

Jäger, Ludwig 2002. Transkriptivität. Zur medialen Logik der kulturellen Semantik. In: Ludwig Jäger und Georg Stanitzek (Hg). Transkribieren Medien/Lektüre. München, Fink: 19-41. 
Jäger, Ludwig 2003. Transkription - zu einem medialen Verfahren an den Schnittstellen des kulturellen Gedächtnisses. TRANS. Internet-Zeitschrift für Kulturwissenschaften. No 15/2003. URL: http://www.inst.at/trans/15Nr/06_2/jaeger15.htm (Stand: 09.03.2014).

Jäger, Ludwig 2004a. Die Verfahren der Medien: Transkribieren - Adressieren - Lokalisieren. In: Jürgen Fohrmann und Erhard Schüttpelz (Hg.). Die Kommunikation der Medien. Tübingen, Niemeyer: 69-79.

Jäger, Ludwig 2004b. Sprache als Medium politischer Kommunikation. Anmerkungen zur Transkriptivität kultureller und politischer Semantik. In: Ute Frevert und Wolfgang Braungart (Hg.). Sprachen des Politischen. Medien und Medialität in der Geschichte. Göttingen: Vandenhoek \& Ruprecht: 323-355.

Kahn, Fritz 1919. Die Zelle. Stuttgart: Franck'sche Verlagshandlung.

Kahn, Fritz 1920. Das Gruseln. Kosmos - Handweiser für Naturfreunde, 12-14.

Kahn, Fritz 1922. Das Gruseln. In: Hanns Günther (Hg.). Wunder in uns. Zürich: Rascher: 249-255.

Kahn, Fritz 1922-1931. Das Leben des Menschen. 5 Bde. Stuttgart: Kosmos, Franckh'sche Verlagshandlung.

Kahn, Fritz 1933. Das Gruseln. In: Kosmos-Taschenkalender für die deutsche Jugend 1933/34: $117-122$.

Kahn, Fritz 1939. Der Mensch gesund und krank. Zürich: A. Müller.

Kahn, Fritz 1940. Der Mensch. Bau und Funktionen unseres Körpers allgemeinverständlich dargestellt. Zürich: A. Müller.

Kahn, Fritz 1943. Big, Blond, Creative, too - The Master Race! Daily Mirror: 10.

Kahn, Fritz 1943a. Man in Structure and Function. New York: Alfred Knopf.

Nemec, Birgit. Anatomical Modernity in Red Vienna: Julius Tandler's Textbook for Systematic Anatomy and the Politics of Visual Milieus. Sudhoffs Archiv, erscheint 2015.

Nikolow, Sybilla und Lars Bluma 2002. Bilder zwischen Öffentlichkeit und wissenschaftlicher Praxis. Neue Perspektiven für die Geschichte der Medizin, Naturwissenschaften und Technik. NTM. Zeitschrift für die Geschichte der Wissenschaften, Technik und Medizin (NF 10): 201-208.

Nikolow, Sybilla und Lars Bluma 2009. Die Zirkulation der Bilder zwischen Wissenschaft und Öffentlichkeit. Ein historiographischer Essay. In: Bernd-Rüdiger Hüppauf und Peter Weingart (Hg.). Frosch und Frankenstein: Bilder als Medium der Popularisierung von Wissenschaft. Bielefeld: Transcript: 45-77.

Mitchell, William J. T. 2008. Das Leben der Bilder. Eine Theorie der visuellen Kultur. München, Beck.

Reichsgesetzblatt vom 7.2.1933. In: documentArchiv.de (Hrsg.), URL: http://www. documentArchiv.de/ns/schutz-dt-vlk.html, (Stand: 09.03.1015).

Sappol, Michael 2006. Dream Anatomy. Bethesda: National Library of Medicine.

Sappol, Michael 2012. The Wonder in Us (1921). In: Michael Sappol, Laura Lindgred und Arne Svenson (Hg.). Hidden treasure: the National Library of Medicine. New York: Blast Books: 160-61.

Sappol, Michael 2013. Radiant modernity: An iconography of rays, beams, and waves, 1920-1960. Vortrag am 08.10.2013 beim Festival of Medical History and the Arts, The New York Academy of Medicine.

Schwarz, Angela 2001. Der Schlüssel zur modernen Welt: Wissenschaftspopularisierung in Großbritannien und Deutschland im Übergang zur Moderne (1870-1914). Stuttgart: Franz Steiner.

Sobotta, Johannes 1904. Atlas der deskriptiven Anatomie des Menschen. I. Abteilung. München: J. F. Lehmann's Verlag.

Stoff, Heiko 2008. Hormongeschichten. Wie sie in den Jahren 1928-1954 von den Wissenschaftsjournalisten Walter Finkler und Gerhard Venzmer erzählt wurden. zeitenblicke 7(3): URL: http://www.zeitenblicke.de/2008/3/stoff (Stand: 09.03.2015).

Venzmer, Gerhard 1930. Hirnmasse und Intelligenz. Kosmos - Handweiser für Naturfreunde: $41-47$.

Venzmer, Gerhard 1930a. Körpergestalt und Seelenanlage. Stuttgart: Franckh'sche Verlagshandlung. 
Venzmer, Gerhard 1933. Deine Hormone - dein Schicksal! Stuttgart: Franckh'sche Verlagshandlung.

Venzmer, Gerhard 1938. Der Mensch und sein Leben. Ein Volksbuch vom menschlichen Körper. Stuttgart: Franckh'sche Verlagshandlung.

Venzmer, Gerhard 1940. Hand- und Lehrbuch der Krankenpflege. Stuttgart: Franckh'sche Verlagshandlung.

Miriam Eilers

Mercator Research Group

"Spaces of Anthropological Knowledge"

Ruhr-Universität Bochum

Universitätsstr. 150

44801 Bochum

Germany

E-Mail: miriam.eilers@rub.de 